\title{
1 Insights into the Human Gut Virome by Sampling a Population from the Indian
}

\section{Subcontinent}

3 Kanchan Bhardwaj ${ }^{\mathrm{a}, \mathrm{b}, *}$, Anjali Garg ${ }^{\mathrm{c}}$, Abhay Deep Pandey ${ }^{\mathrm{a} \#}$, Himani Sharma ${ }^{\mathrm{a} \#}$, Manish Kumar

4 and Sudhanshu Vrati ${ }^{\mathrm{a}}{ }^{*}$

$5 \quad$ Regional Centre for Biotechnology, NCR Biotech Science Cluster, Faridabad-Gurugram

6 Expressway, Faridabad-121 001, Haryana, India.

$7 \quad{ }^{b}$ Manav Rachna International Institute of Research and Studies, Sector-43, Aravali hills,

8 Faridabad-121004, Haryana, India.

$9{ }^{c}$ Department of Biophysics, University of Delhi South Campus, New Delhi-110021, India.

$10 \quad{ }^{\#}$ Equal contributors.

$11 *$ Corresponding authors:

12 Kanchan Bhardwaj

13 kanchan.fet@mriu.edu.in

14 Sudhanshu Vrati

15 vrati@rcb.res.in

16

17 Keywords: virus-like-particles; total microbial DNA; metagenomics; gut virome; temperate;

18 crAss-like phages; Indian subcontinent 


\section{Abstract}

25 Gut virome plays an important role in human physiology but remains poorly understood. This

26 study reports an investigation of the human gut DNA-virome of a previously unexplored ethnic

27 population through metagenomics of faecal samples collected from individuals residing in

28 Northern India. Analysis shows that, similar to the populations investigated earlier, majority of

29 the identified virome belongs to bacteriophages and a smaller fraction $(<20 \%)$ consists of

30 viruses that infect animals, archaea, protists, multiple domains or plants. However, crAss-like

31 phages, in this population, are dominated by the genera VII, VIII and VI. Interestingly, it also

32 reveals the presence of a virus family, Sphaerolipoviridae, which has not been detected in the

33 human gut earlier. Viral families, Siphoviridae, Myoviridae, Podoviridae, Microviridae,

34 Herelleviridae and Phycodnaviridae are detected in all of the analyzed individuals, which

35 supports the existence of a core virome. Lysogeny-associated genes were found in less than $10 \%$

36 of the assembled genomes and a negative correlation was observed in the richness of bacterial

37 and free-viral species, suggesting that the dominant lifestyle of gut phage is not lysogenic. This

38 is in contrast to some of the earlier studies. Further, several hundred high-quality viral genomes

39 were recovered. Detailed characterization of these genomes would be useful for understanding

40 the biology of these viruses and their significance in human physiology.

\section{Importance}

43 Viruses are important constituents of the human gut microbiome but it remains poorly

44 understood. The Indian subcontinent is a unique biogeographic region and the Indian population

45 is known to harbour a distinct bacterial microbiome. However, the gut virome in this population 
46 has not been investigated earlier. Therefore, in this study, we investigated fecal samples of 12

47 healthy individuals to analyze their gut virome, through metagenomics.

\section{Introduction}

The human gut harbours a microbial ecosystem that consists of trillions of microbes

51 including bacteria, viruses, fungi, archaea and protists. ${ }^{1}$ Majority of the microbiome studies

52 have focused on the bacterial component. Our understanding of the virome has been

53 hampered, primarily due to technical challenges associated with analysis of viruses as well

54 as due to limited availability of resources such as comprehensive viral databases and virus-

55 specific tools for bioinformatic analyses. ${ }^{2}$ Nevertheless, metagenomics of faecal samples

56 has provided significant insights into virome composition, led to discovery of novel

57 viruses, revealed association with diseases and ethnic populations, and contributed to

58 development of viral databases..$^{3-10}$ Although, most of the data generated by metagenomics

59 studies remains uncharacterized (86-99\% of the sequencing reads) and no consensus has

60 emerged on the existence of a core gut virome, the identifiable fraction is reported to be

61 dominated by DNA-bacteriophages in all studies. ${ }^{7,11}$ Hence, in addition to understanding

62 the viral composition, it has also been of interest to understand the dynamics between

63 bacteriophages and bacteria in this ecosystem. Towards this, initial studies suggested that

64 the human gut is dominated by temperate phages unlike other microbe-rich ecosystems

65 such as the oceans. ${ }^{12-15}$ Although, a recent analysis by Shkoporov et al. ${ }^{16}$ has indicated that

66 temperate bacteriophages do not dominate the human gut. ${ }^{16}$ Hence, further investigations 
67 are still required for establishing the nature of microbial dynamics in the gut as well as to

68 address if there is existence of any core virome.

69 Several studies showing an association of gut virome with diseases as well as

70 engraftment of phages in successful FMT trials have indicated the possibility of diagnostic

71 and therapeutic use of gut phageome. ${ }^{9,10,12,18,19}$ However, for translational application of the

72 virome, it would be important to establish the impact of various factors such as age, genetic

73 background and geography on the variation of healthy virome. ${ }^{16}$

74 The Indian subcontinent represents a unique biogeographic region and the Indian

75 population is known to harbour a distinct bacterial microbiome with respect to its diversity

76 and the prevalent microbial taxa. ${ }^{20}$ It is possible that it will have a characteristic co-residing

77 virome. Therefore, we investigated the gut virome by sampling a population from the

78 Indian subcontinent. We analysed the DNA viruses present in the faecal samples of 12

79 "healthy" individuals by shotgun sequencing of the DNA isolated from purified virus-like-

80 particles (VLPs) as well as from total microbial populations. Here, we present the

81 composition of "healthy Indian gut virome" and demonstrate the presence of a virus

82 family, Sphaerolipoviridae, which has not been previously reported in the human gut. We

83 also demonstrate how the choice of sample preparation can impact virome analysis.

84 Further, this study provides insights into the phylogenetic core gut virome and the viral

85 lifestyle in the gut.

$87 \quad$ Results

88 (i) Effect of sample preparation on virome analysis

89 There are two methods for the isolation of viral DNA from fecal samples. One involves 
enrichment of virus-like-particles (VLPs) followed by extraction of the VLP-DNA. The second

91 method involves extraction of total microbial DNA without enrichment for VLPs. In most of the

92 earlier analyses of fecal viromes, one of the two methods has been used. There is only one recent

93 study by Shokoporov et al. (2019) where both methods were used. ${ }^{16}$ Here, we also used both

94 methods to extract DNA from each faecal sample to include free-viruses as well as host-

95 associated-viruses in our analysis. Shotgun sequencing of the VLP-DNA and the total-microbial

96 DNA generated 20-150 million reads per sample. The raw reads were filtered to remove low-

97 quality sequences as well as any contaminating sequences of human origin (Figure 1A). We note

98 that the recovery of high-quality sequences varied between samples although, equal starting

99 material was used for each sample and the samples were processed similarly (Figure 1A).

100 Further, mapping of the quality-filtered reads to the NCBI viral RefSeq database showed

101 alignment of $0.2 \%$ of the VLP-DNA-derived and $0.05 \%$ of the total microbial DNA reads

102 (Table S1). Notably, only a limited fraction of the total reads could be mapped to the viral

103 reference database, which is similar to what has been observed in earlier studies and has been

104 attributed to the limited size of the reference databases. Nevertheless, the proportion of viral

105 sequences in the VLP-DNA fractions was higher than in the total microbial DNA fractions

106 (Table S1). Next, we analysed the read quality with the tool, ViromeQC. This tool is

107 programmed to assign enrichment scores to sequences, based on their alignment to non-viral

108 genes including small subunit and large subunit ribosomal RNA genes, and single-copy markers.

109 Strikingly, enrichment scores assigned to the VLP-DNA-derived reads were significant $(\geq 5.0)$ as

110 well as higher than the scores assigned to the total microbial DNA-derived reads, indicating that

111 the VLP fractions were significantly enriched for viral sequences and were free of contamination

112 by the host DNA (Figure 1B). We again note that despite similar processing, there is variation 
among samples (Figure 1B).

Following metagenome assembly of the quality-filtered reads, we recovered around 6-fold compared to the VLP-DNA fractions (93,612 contigs) (Table S2). This is possibly because in most samples we had recovered more number of high-quality raw reads from the total microbial DNA

118 fractions as compared to the VLP-DNA fractions (Table S2). We analysed these contigs for their 119 functional profiles, using the SEED subsystems database on the MG-RAST platform. Analysis 120 revealed that the most abundant category of functions encoded by the VLP-DNA-derived contigs 121 was "phages, prophages, transposable elements and plasmids" (13-20\%) followed by the 122 "clustering-based subsystems" (12-15\%) (Figure 1C). The "clustering-based subsystems" 123 represent those proteins for which the exact roles are not yet known. Whereas, functional profiles 124 of the total microbial DNA-derived contigs showed a relatively higher proportion of genes related 125 to "stress response", "respiration", "nucleosides and nucleotides", "amino acids and derivatives" 126 but significantly lower proportions of "clustering-based subsystems" $(<4 \%)$ and "phages, 127 prophages, transposable elements and plasmids" functions $(<3 \%)$ (Figure 1C). Detection of high 128 levels of genes related to "phages, prophages, transposable elements and plasmids" further 129 confirmed that VLP purification resulted in enrichment of viral sequences. This analysis also 130 highlights that a significant proportion of viral genes encode for proteins with unknown functions. For a more stringent selection of viral sequences from the total pool of contigs, we used

132 three virus-mining tools, VirFinder, VirSorter and CAT. Notably, VirFinder identified a greater 133 number of contigs as viral in the total microbial DNA fractions as compared to the VLP-DNA134 derived fractions (Table S3). Whereas, VirSorter and CAT identified more number of contigs as 135 viral in the VLP-DNA-derived fractions (Table S3). In total, around $35.7 \%$ of the VLP-DNA- 
136 derived contigs $(>1 \mathrm{~kb})$ and around $7.3 \%$ of the total microbial DNA-derived contigs $(>1 \mathrm{~kb})$ were

137 identified as viral by the three tools (Table S3). The VLP-DNA- and the total-microbial DNA-

138 derived viral contigs were pooled for individual samples and redundancy was removed within each

139 sample. In total, we recovered 61,099 viral contigs ( $>1 \mathrm{~kb})$, of which $36.6 \%$ were derived from

140 VLP DNA and 63.4\% were from total microbial DNA (Table S3).

141 Next, we used the tool CheckV to examine the quality of the identified viral sequences.

142 CheckV categorized the contigs as "complete genomes", "high-quality" (more than $90 \%$ complete

143 genomes), "medium-quality" (50-90\% complete genomes), "low-quality" (0-50\% complete

144 genomes) and "undetermined quality" (Figure 1D; Table S4). It determines completeness by

145 comparing query sequences with the database of complete viral genomes followed by estimation

146 of expected genome sizes. Genomes are categorized as "complete", based on the presence of the

147 direct terminal repeat (DTR) or inverted terminal repeat (ITR) sequences or provirus integration

148 sites. Sequences are categorized as "undetermined quality" when they do not match any of the

149 CheckV reference genomes and do not have any viral hidden Markov models (HMMs). Therefore,

150 contigs that are classified as "undetermined quality" could represent very novel viruses, very short

151 contigs or they may not be viral at all. In total, we recovered 600 "complete" viral genomes, out

152 of which 437 were from VLP-DNA and 163 from total microbial DNA. Overall, these results show

153 that viral sequences could be obtained with or without VLP enrichment. However, the sequences

154 recovered by each method are unique. Further, the method of VLP enrichment before DNA

155 extraction resulted in better recovery of "complete genomes". 
(ii) Identification of known and unknown families of viruses

159 bacteriophages $(84.8 \pm 17.6 \%)$, animal viruses $(4.29 \pm 2.8 \%)$, protist viruses $(2.3 \pm 3.17 \%)$,

160 archaeal viruses $(0.11 \pm 0.12 \%)$, plant viruses $(0.1 \pm 0.2 \%)$ and viruses that infect multiple

161 domains $(1.29 \pm 1.1 \%)$ (Figure $2 \mathrm{~A})$. Further, we annotated the viral contigs, either based on

162 sequence alignment or by clustering of their predicted proteins. Protein alignment-based

163 program, Kaiju, assigned taxonomic classification to $\sim 18 \%$ of the total contigs $(10,917$ contigs

164 out of the total 61,099) (Figure 2B). The 13 identified bacteriophage families $(9,832$ contigs),

165 include Siphoviridae, Myoviridae, Podoviridae, Herelleviridae, Autographviridae,

166 Ackermannviridae, Microviridae, Demerecviridae, Drexlerviridae, Tectiviridae, Chaseviridae

167 and Inoviridae. The identified animal viruses (141 contigs) were from 13 families,

168 Herpesviridae, Iridoviridae, Ascoviridae, Poxviridae, Malacoherpesviridae, Circoviridae,

169 Polyomaviridae, Alloherpesviridae, Asfarviridae, Adenoviridae, Lavidaviridae,

170 Papillomaviridae and Parvoviridae. Further, 518 contigs were assigned to a single-family

171 Phycodnaviridae; 406 contigs as protist viruses of two families, Mimiviridae and

172 Marseilleviridae; 17 Archaea viruses of two families, Pithovirus sibericum and

173 Sphaerolipoviridae; 2 viruses of the Genomoviridae family, and 3 plant viruses of the

174 Caulimoviridae family (Figure 2B). Similar to virome analyses in other ethnic groups, we note

175 that the majority of the identifiable reads as well as the assembled genomes belong to

176 bacteriophages. Although mapping to protozoan, invertebrate and plant viruses has been

177 observed in earlier studies as well, their relationship with the human gut has not been proven. ${ }^{21}$

178 Additionally, it has been suggested that mapping to large dsDNA genomes of Ascoviridae, 179 Iridoviridae, Marseilleviridae, Mimiviridae, Nudiviridae, Phycodnaviridae, Pithovirus and 
180 Poxviridae families could be due to misassignments. ${ }^{21}$ Therefore, we also used the protein 181 clustering-based method for taxonomic annotation.

Protein clustering was done with vConTACT2 and the clusters were identified with the

183 help of two databases i.e. TrEMBL and ProkaryoticViralRefSeq94-Merged. Fifteen families

184 were identified when the TrEMBL database was used for taxonomy assignment (Figure 2C).

185 Except for Baculoviridae, Bicaudaviridae and Nudiviridae, all other families identified by this

186 method were also identified by the protein-alignment-based method (Figure $2 \mathrm{~B}$ ). Among the

187 animal viruses, Herpesviridae was detected by both methods. However, a significant proportion

188 of the clusters remained unassigned (Figure 2C). The use of the ProkaryoticViralRefSeq94-

189 Merged database resulted in the identification of 20 families of the prokaryotic viruses (Figure

190 2D).

191 Altogether, sixteen families including, Siphoviridae, Myoviridae, Podoviridae,

192 Herelleviridae, Microviridae, Inoviridae, Ackermannviridae, Phycodnaviridae, Mimiviridae,

193 Sphaerolipoviridae, Tectiviridae, Circoviridae, Genomoviridae, Iridoviridae, Herpesviridae and

194 Poxviridae were identified by both, protein alignment as well as protein clustering-based

195 methods (Figure 2B, 2C and 2D).

196 We also noted that the reads from all of our samples except one mapped to crAss-like

197 phages (0.1-25\% of the total reads) (Table S5). Among the assembled metagenomes, we identified

198 a total of 382 putative crAss-like phages (contigs $>70 \mathrm{~kb}$ long), based on their sequence homology

199 to seven most conserved proteins of crAss-like phages (Table S5). Around $30 \%$ of these contigs

200 could be classified into the ten known crAss-like phage genera, with VI, VII and VIII being the

201 most dominant ones (Figure 2E). Further, around 43\% of the identified crAss-like phages were 
202 found to contain at least one of the five lysogeny-related genes i.e. transposase, integrase, 203 excisionase, resolvase and recombinase (Table S5).

(iii) Evidence for the existence of a core virome

Although most of the viruses that are found in the human gut are unique to an individual,

207 there are suggestions of a "core virome" or part of virome that is shared by individuals.

208 However, its identity and the extent of sharing have varied in different studies, leaving the

209 concept of a "core virome" debatable at this time. ${ }^{12,15,16,22}$ Here, based on the protein alignment

210 method, we found that 9 families out of 32 were present in all individuals (Figure 3A). These

211 include Siphoviridae, Myoviridae, Podoviridae, Phycodnaviridae, Herelleviridae, Mimiviridae,

212 Microviridae, Demerecviridae and Herpesviridae. When using the protein clustering-based

213 method, the shared clusters identified with the TrEMBL database were Siphoviridae,

214 Myoviridae, Podoviridae, Microviridae and Phycodnaviridae (Figure 3B). Whereas, the shared

215 clusters identified with the ProkaryoticViralRefSeq94-Merged database belong to families

216 Siphoviridae, Myoviridae, Podoviridae, Microviridae, Inoviridae, Herelleviridae,

217 Ackermannviridae, Rudiviridae, Fuselloviridae, Leviviridae, Sphaerolipoviridae, Tectiviridae,

218 Cystoviridae, Bicaudaviridae, Lipothrixviridae, Pleolipoviridae, Ampullaviridae, Corticoviridae,

219 Globuloviridae, Turriviridae and Phycodnaviridae (Figure 3C). Collectively, the 6 families that

220 were found shared among all individuals by both methods include Siphoviridae, Myoviridae,

221 Podoviridae, Microviridae, Herelleviridae, and Phycodnaviridae. 
(iv) Lifestyles of the gut-resident bacteriophages and its effects on the co-residing bacterial

224

225

226

227

228

229

230

231

232

233

234

235

236

237

238

239

240

241

242

243

244

245

\section{population}

The majority of the identified viruses in the gut are bacteriophages, which can significantly influence the structural and functional output of the ecosystem through processes such as host predation, lysogeny and horizontal gene transfer. Towards understanding their interaction with the co-residing bacterial population, we determined the richness of the bacterial as well as the viral species derived either from VLP-DNA or from total microbial DNA fractions (Figure 4A). The analysis revealed a moderate negative correlation between the richness of bacterial species and the VLP-DNA-derived viral species $(\mathrm{r}=-0.6 ; \mathrm{p}=0.037)$ but no significant correlation was observed with the total microbial DNA-derived viral species $(r=-0.12 ; \mathrm{p}=0.71)$ (Figure 4B). Generally, VLPs (free virus particles) represent those viruses, which are undergoing lytic cycles. Whereas, total microbial DNA-associated viruses represent those, which are in a lysogenic state with the host; either integrated into the host genomes or existing as an extrachromosomal entity such as plasmids. Our results showing a negative correlation between bacterial and free viral (lytic) species richness align with the idea that dominance by phages that are undergoing lytic lifecycle results in reduced bacterial abundance. Further, we observed no correlation between the richness of bacterial species and the total microbial DNA-derived viral species (lysogenic). This is an unexpected result because an increase in the abundance of the bacterial population would be expected to increase the abundance of lysogenic phages. However, no correlation is possible if the number of lysogens present in the analysed population were low.

Therefore, we determined the proportion of lysogenic viruses in our population by scanning our vOTUs with HMM profiles of five lysogeny-associated genes (transposase, integrase, excisionase, resolvase and recombinase). The vOTUs were assigned lysogenic if at least one of 
246 these lysogeny-associated genes was present in them. Across all individuals, we found that less

247 than $10 \%$ of the vOTUs contained lysogeny-associated genes (Figure 5A). Further, majority of

248 the lysogenic vOTUs were derived from the total microbial DNA fractions (40-97\%) followed

249 by the VLP fractions (3-57\%) and some were represented in both fractions (0-11\%) (Figure 5B).

250

251 Discussion

The process of sample preparation for virome analysis is a significant

253 consideration. In most of the previous metagenome analyses of human gut virome, samples

254 had been prepared either by the enrichment of the VLPs before DNA extraction or by

255 extraction of the total microbial DNA without enrichment. There is only one study where

256 both methods were used to analyse 10 samples. Extraction of the total microbial DNA

257 without enrichment is attractive and could be preferred for multiple reasons such as it

258 provides convenience, is more economical, faster and can be adapted for simultaneous

259 processing of a large number of samples. However, it is important to establish the effects

260 of sample preparation. To address this, Gregory et al. ${ }^{7}$ performed bioinformatic analysis of

261 the available metagenome data, collected in different gut virome studies. Their analysis

262 revealed that (i) there is no significant effect of the two methods on the number of viral

263 contigs assembled per bp sequenced; (ii) sample preparation method also showed no effect

264 on the contig length, when data from different studies were analysed but longer contigs

265 were detected with VLP-DNA-derived metagenomes when data from a single study which

266 used both methods, were compared; (iii) the rate of viral detection was higher with the data

267 obtained from the total microbial DNA extraction method; and (iv) the two methods

268 capture different subsets of viruses. ${ }^{7}$ Similarly, our results also show that each method 
recovers a comparable number of viral contigs and that the viral sequences captured by the

270 two methods are unique. In addition, we found that the recovery of "complete viral

271 genomes" is better with the VLP enrichment method. This could be because the VLP-DNA

272 fractions are significantly enriched with sequences of viral origin, which possibly led to

273 better genome assembly. Our analysis also shows that the total microbial DNA fraction is

274 enriched with phages containing lysogeny-associated genes, although not all. Therefore,

275 data obtained through the total microbial DNA method could be useful for understanding

276 the mechanisms related to the co-existence of phages with their hosts. However,

277 concurrent analysis by both methods would be needed for a comprehensive understanding

278 of a virome.

279 Taxonomic assignment of our data reveals that the gut virome is dominated by

280 bacteriophages, which is similar to what has been observed in other populations. Although,

281 one of the viral families, Sphaerolipoviridae, found in our samples has not been reported in

282 the human gut earlier. Bacteriophages of the Herelleviridae family have been reported in

283 one of the recent studies involving cohorts of Chinese and migrant Pakistani populations. ${ }^{24}$

284 Strikingly, we did not detect viruses of the Anelloviridae family, which are the

285 predominant animal viruses in the Western population. ${ }^{7}$ The most abundant animal virus

286 family in our samples was Herpesviridae. Based on the analysis by multiple methods, we

287 found that members of at least six families, Siphoviridae, Myoviridae, Podoviridae,

288 Microviridae, Herelleviridae, and Phycodnaviridae were present in all individuals. Since

289 this analysis is based on a small fraction of identifiable sequences of the whole virome, it is

290 not possible to estimate the extent of sharing between individuals. However, it does

291 provide support for the existence of a phylogenetic core virome. Families of the order 
292 Caudovirales (Siphoviridae, Myoviridae, Podoviridae) and Microviridae have been known

293 to form a phylogenetic core phageome..$^{3,15,22}$ Interestingly, we found that in addition to these

294 phage families, members of the Herelleviridae and Phycodnaviridae families were present

295 at significant levels and were shared among all individuals. Herelleviridae is a relatively

296 new family of phages added to the order Caudovirales. Viruses of the Phycodnaviridae

297 family are large dsDNA viruses that are known to infect algae and there are pieces of

298 evidence that they can infect humans as well. ${ }^{25}$ Further, we detected crAss-like phages in

299 all except one sample. Since the discovery of crAssphage in 2018, the family of crAss-like

300 phages has gained special interest because they are known as the most abundant phages in

301 the human gut and are quite ubiquitous as well. ${ }^{26}$ In some of the gut viromes, up to $90 \%$ of

302 the sequences are comprised of crAss-like phages. They have been classified into 4

303 subfamilies and 10 genera. ${ }^{23,27}$ Using similar methods, we were able to classify only $30 \%$

304 of our crAss-like phages, with genera VI, VII and VIII being the most represented. In the

305 Western population, the most common genus is I and in the Malawian cohorts, they are

306 genera VIII and IX. The significant fraction that remains unclassified requires further

307 investigation.

308 Bacteriophages are important components of the gut ecosystem and therefore,

309 understanding the mechanism(s) of phageome maintenance in the gut has been of interest.

310 Apart from environmental conditions of the gut as well as the host-defense and phage

311 counter defense system, phage lifestyle plays a significant role in this. However, consensus

312 about the phage lifestyle in the human gut has not been reached. Our results showed a

313 negative correlation in the richness of bacterial species and the VLP-derived viral species.

314 VLP-derived viruses represent those viruses, which are produced upon the lytic cycle. 
315 These results, therefore, demonstrate the effect of the lytic lifestyle of phages on the co-

316 residing bacterial population in the gut. Further, we detected lysogeny-associated genes

317 only in a small fraction of viral sequences $(<10 \%)$. These results further suggest that the

318 lysogenic lifestyle does not dominate in the gut. Many of the earlier investigations of gut

319 virome have suggested that lysogenic lifestyle dominates in the gut. ${ }^{12,15,17}$ However, our

320 results corroborate with one of the recent studies, which also reported that temperate

321 phages do not dominate the gut ecosystem. ${ }^{16}$ Similarly, although crAss-like phages were

322 initially predicted to have temperate lifestyle, evidences are emerging to suggest the

323 existence of alternative lifestyles..$^{23}$ In our analysis, we found lysogeny-associated genes in

$32443 \%$ of the crAss-like phages. All these results suggest that in addition to lytic and

325 lysogenic lifestyles, alternative phage lifestyles such as pseudolysogeny, chronic infection

326 and carrier state might be operative for the maintenance of phages and their hosts in the gut

327 ecosystem. However, further investigations will be needed to fully understand their

328 existence and contribution.

329 This study has generated data on the virome comprising only the DNA containing

330 viruses. To produce a complete picture of the "healthy Indian gut virome" attempts are

331 underway to identify the RNA-genome containing resident viruses of the gut. The

332 recovered "complete genomes" are also being analyzed further according to the guidelines

333 provided by the Genomic Standards Consortium for reporting the sequences of

334 uncultivated viral genomes (UViG). 

Committees (Reference No. RCB-IEC-H-14), recruited pre-defined "healthy" individuals, and collected faecal samples from them after obtaining written consent. Individuals between the ages of 20 and 35 years, who had normal body mass index, normal bowel frequency, no history of

342 chronic intestinal disease or autoimmunity, had balanced meals at regular intervals, and had not 343 received antibiotics in the 6 months before sampling were defined as "healthy". Samples were

344 collected using sterile containers, placed on ice immediately after collection, and then stored in a deep freezer, to be used within 3 months of collection.

\section{DNA extraction and sequencing:}

VLP purification was done by sequential centrifugation, filtration and gradient ultracentrifugation. Homogenized extracts of the faecal samples were prepared by dilution of specimens in ice-cold SM (sodium and magnesium) buffer followed by thorough vortexing in presence of glass beads. Extracts were centrifuged at $4500 \mathrm{~g}$ for $30 \mathrm{~min}$ at $4{ }^{\circ} \mathrm{C}$, to remove

352 particulate material. The supernatants were filtered sequentially through $0.45 \mu \mathrm{m}$ and $0.22 \mu \mathrm{m}-$ 353 pore-size membranes. The filtrates were centrifuged on a step gradient of iodixanol. Before 354 isolation of DNA from the purified VLPs, we treated them with DNase I and Benzonase to remove 355 any free nucleic acid of human and bacterial origin that may co-purify during our procedure. To confirm the removal of contaminating bacterial DNA, VLP preparations were screened by $16 \mathrm{~S}$

357 rDNA PCR. DNA was extracted from those VLP preparations that resulted in no amplification of 358 DNA fragments with primers targeted for 16S rDNA (data not shown). VLP-DNA was extracted 359 using the phenol:chloroform method. For the extraction of total microbial DNA, homogenized 
361 thiocyanate to lyse microbial cell walls and membranes followed by mechanical disruption using

362 a bead beater. Insoluble fractions were removed by centrifugation and the total microbial DNA

363 was isolated from the supernatants through conventional methods of nucleic acid precipitation.

364 RNA was removed by digestion with RNaseA. ${ }^{28}$ Purified DNA was quantitated and its quality was

365 checked before sequencing. Whole-genome shotgun sequencing was performed for all samples.

366 Whole-genome metagenome sequencing libraries were prepared using the TruSeq DNA PCR-Free

367 library preparation kit. Briefly, DNA was sheared using Covaris ultra sonicator. The fragmented

368 DNA was end-repaired to remove any overhangs resulting from sonication. Library size was

369 selected using sample purification beads. The size-selected molecules were mono-adenylated at

370 the 3'-end followed by ligation of Illumina indexed adapters. The adapter-ligated fragments were

371 cleaned up using purification beads and the clean fragments were assessed for size distribution on

372 Agilent TapeStation. To include ssDNA viruses in the analysis, VLP DNA was PCR-amplified

373 before shotgun sequencing, using Swiftbio kit from Illumina. In brief, whole-genome metagenome

374 sequencing libraries were prepared using Accel-NGS 1S Plus DNA Library Kit, which processes

375 both ssDNA and dsDNA in a mixed sample type. DNA was sheared using Covaris ultra sonicator

376 and then denatured for adaptase step wherein 3' tailing and ligation of truncated adapter 1 takes

377 place. Next, the second strand of DNA is generated followed by truncated adapter 2 ligation in an

378 extension and ligation step. The fragments were indexed using a limited cycle PCR, cleaned up

379 using purification beads, and assessed for size distribution using Agilent TapeStation. The

380 resulting libraries were quantitated and loaded on the cBot for cluster generation. Sequencing was

381 performed on Illumina HiSeq X Ten platform with a read length of 150x2 bp. 
The raw reads were processed using fastq-mcf (v1.1.2) to ensure that the data do not contain sequencing artifacts $\left(\mathrm{Q}_{30}\right)$, sequence duplication and adapter sequences. Reads with an average quality Phred score below 30, low-quality tails in the reads and reads shorter than 36 bases were eliminated. Further, the high-quality reads were also filtered for human DNA contamination by aligning the reads to the human reference genome (hg19/GRCh37) using the Burrows-Wheeler aligner (BWA) and only the unaligned reads were taken for further processing. Filtered total microbial DNA reads were then aligned to bacterial, fungal, viral and archaea genomes in the NCBI RefSeq database. To evaluate the purity of the VLP samples, ViromeQC (v1.0.1) was used. ${ }^{29}$ Metagenome assembly was performed with MEGAHIT (v 1.2.9).

\section{Identification of viral sequences:} sequences using tools Virfinder (v1.1) and Virsorter v2. Virsorter v1 was used for the identification

397 of viral sequences obtained from the total microbial DNA samples. Extraction of viral contigs with 398 Virfinder was based on a score of $>0.7$ and $p$-value $<0.05$. Virsorter 2 viral contigs were considered with a cut-off score of $>0.75$. For the bacterial samples, Virsorter-identified contigs belonging to all categories (categories 1-6) were considered for further downstream analysis. All the "non-viral" 401 contigs from Virfinder and Virsorter were taken as input for CAT (Contig Annotation Tool, v5.2). 402 All the viral contigs obtained by the above three tools (VirFinder, VirSorter and CAT) were sorted 403 for each of the 12 samples individually and the collection was run through the CD-HIT-EST 404 (v4.8.1) tool with an identity cut off of 99\% over the entire contig length to get non-redundant 405 viral databases for each sample. To analyze the quality of assembled genomes, CheckV (v0.6) tool 406 was used. ${ }^{30}$ 
For taxonomic identification of the viral contigs, an index was made from Viral RefSeq database 1.1, 2.1 and 3.1 (https:/ftp.ncbi.nlm.nih.gov/refseq/release/viral/), using Bowtie2. The

409 Kaiju program was then used to map the contigs to the Viral RefSeq index. For the clustering410 based method of taxonomic identification, genes/ORFs were predicted for each of the 12 viral

411 databases using the Prodigal (v2.6.3) tool in metagenomic mode. vConTACT2 was used to

412 cluster and provide a taxonomic context of metagenomic sequencing data. Prodigal GenBank

413 coordinates were used as input for each sample for vConTACT2 and it was run with pc-inflation

414 and vc-inflation set to 1.5 , pcs-mode set to MCL, and vcs-mode set to ClusterONE. ${ }^{31}$ Databases

415 used for the analysis were TrEMBL and ProkaryoticViralRefSeq94-Merged.

\section{5. crAssphage analysis}

To identify crAss-like phages, all assembled metagenomic contigs were BLAST (v2.4.0)

419 searched against a database of crAssphage genomes and proteomes. The database comprised of 420 prototypical crAssphage genome and proteome (p-crAssphage; NC_024711.1), 249 crAssphage

421 genome and 2684 crAssphage family, which were reported by Guerin et al., and Yutin et al.,

422 respectively. ${ }^{23,27}$ From the search result, putative crAss-like phages were selected using the

423 following criteria (i) a BLAST hit against databases with an E-value less than 1E-05, (ii) a BLAST

424 query alignment length $350 \mathrm{bp}$, and (iii) a minimum contig length of $70 \mathrm{~kb}$ (representing near-

425 complete crAss-like phage contigs).

426 To determine the phage lifestyle, open reading frames (ORFs) for each crAss-like phage 427 contigs were predicted using Prodigal (v2.6.3) in metagenomic mode

428 (https://github.com/hyattpd/Prodigal). Further, all ORFs were compared to a custom set of 29 429 HMM profiles that belong to transposase, integrase, excisionase, resolvase and recombinase 
430 proteins. The HMM profiles were downloaded from the Pfam database. ${ }^{32}$ Contigs with an ORF,

431 which obtained a hit with any of the above mentioned five functional classes were classified as

432 temperate. ${ }^{33}$

433 The taxonomic identity of predicted crAss-like phages was done based on average 434 nucleotide identity (ANI) calculated using OrthoANI at default parameters. ${ }^{34}$ ANI of each 435 predicted crAss-like phages were calculated with each of the sixty-five previously identified 436 crAss-like phages genomes classified as genus I to X. ${ }^{35}$ The genus having maximum ANI was 437 assigned as the most probable taxon.

\section{Determination of species richness and their correlations:}

440 Quality filtered reads obtained from the VLP-derived DNA and the total microbial DNA were

441 mapped to a Viral RefSeq index, using ViromeScan2. The index was made from the collection of

442 eukaryotic viruses and bacteriophages present in the Viral RefSeq database using Bowtie2. The

443 output files were mapped to the NCBI taxa accession number using a custom code and an OTU

444 table was generated. Similarly, reads obtained from total microbial DNA were mapped to the

445 bacterial RefSeq database to estimate the abundance of bacterial species, using the MEGAN5

446 package. A vegan package was applied to calculate the richness of viral and bacterial species in

447 each sample.

\section{Functional profiles:}

450 The non-redundant databases of our viral contigs $(>1 \mathrm{~kb})$ from each sample were submitted to

451 the web-server, MG-RAST (Metagenomics Rapid Annotations using Subsystems Technology) 
452 (http://rast.nmpdr.org). The parameters used for the assignment to functional categories were e-

453 value 1e-05, \% identity cut-off of $60 \%$ and minimum alignment length 15.

454

\section{8. Determination of bacteriophage lifestyle}

456 Viral sequences were clustered into vOTUs using ClusterGenomes v5.1 at $95 \%$ average

457 nucleotide identity (ANI) and $85 \%$ average fraction (AF). ORF in each vOTU were predicted

458 using Prodigal v2.6.3 (https://github.com/hyattpd/Prodigal) in metagenomic mode. All ORFs of

459 each OTU were annotated using a custom set of 29 HMM profiles belonging to transposase,

460 integrase, excisionase, resolvase, and recombinase proteins, using the library of $29 \mathrm{HMM}$

461 profiles that was originally compiled by Cook et al. from the Pfam database. ${ }^{33}$

\section{References}

463 1. Matijašic'M, Meštrovic' T, Paljetak HC, Peric' M, Bareši'c A, and Verbanac D. Gut

464 Microbiota beyond Bacteria-Mycobiome, Virome, Archaeome, and Eukaryotic

465 Parasites in IBD. Int. J. Mol. Sci. 2020; 21: 2668-2688. doi:10.3390/ijms21082668.

$466 \quad$ PMID: 32290414.

2. Shkoporov AN, Hill C. Bacteriophages of the Human Gut: The "Known Unknown" of the Microbiome. Cell Host \& Microbe. 2019; 25 (2): 195-209. doi: 10.1016/j.chom.2019.01.017. PMID: 30763534.

3. Guerin E, Hill C. Shining light on human gut bacteriophages. Frontiers in Cellular and Infection Microbiology. 2020; 10:481. doi: 10.3389/fcimb.2020.00481. PMID: 33014897

472 4. Rampelli S, Schnorr SL, Consolandi C, Turroni S, Severgnini M, Peano C, Brigidi P, 473 Crittenden AN, Henry AG, Candela M. Metagenome Sequencing of the Hadza HunterGatherer Gut Microbiota. Curr Biol. 2015; 25(13):1682-1693. doi: 
10.1016/j.cub.2015.04.055. PMID: 25981789. bacteriophage discovered in the unknown sequences of human faecal metagenomes. Nat Commun. 2014; 5: 4498. doi: 10.1038/ncomms5498. PMID: 25058116. expansion of human gut bacteriophage diversity. Cell. 2021; 184(4): 1098-1109. doi: 10.1016/j.cell.2021.01.029. PMID: 33606979.

7. Gregory AC, Zablocki O, Zayed AA, Howell A, Bolduc B, Sullivan MB. The Gut Virome Database Reveals Age-Dependent Patterns of Virome Diversity in the Human Gut. Cell Host Microbe. 2020;28(5):724-740. doi: 10.1016/j.chom.2020.08.003. PMID: 32841606.

8. Paez-Espino D, Roux S, Chen IA, Palaniappan K, Ratner A, Chu K, Huntemann M, Reddy TBK, Pons JC, Llabrés M, Eloe-Fadrosh EA, Ivanova NN, Kyrpides NC. IMG/VR v.2.0: an integrated data management and analysis system for cultivated and environmental viral genomes. Nucleic Acids Res. 2019;47(D1):D678-D686. doi: 10.1093/nar/gky1127. PMID: 30407573.

9. Monaco CL, Gootenberg DB, Zhao G, Handley SA, Ghebremichael MS, Lim ES, 
10. Norman JM, Handley SA, Baldridge MT, Droit L, Liu CY, Keller BC, Kambal A, Monaco CL, Zhao G, Fleshner P, Stappenbeck TS, McGovern DP, Keshavarzian A, Mutlu EA, Sauk J, Gevers D, Xavier RJ, Wang D, Parkes M, Virgin HW. Diseasespecific alterations in the enteric virome in inflammatory bowel disease. Cell. 2015;160(3):447-460. doi: 10.1016/j.cell.2015.01.002. PMID: 25619688. analysis of composition and dynamics. Mob DNA. 2017;8:12. doi: 10.1186/s13100-0170095-y. PMID: 29026445. the faecal microbiota of monozygotic twins and their mothers. Nature. 2010;466(7304):334-338. doi: 10.1038/nature09199. PMID: 20631792. viruses in human feces. Appl Environ Microbiol. 2011;77(22):8062-8070. doi: 10.1128/AEM.06331-11. PMID: 21948823. human gut virome: inter-individual variation and dynamic response to diet. Genome Res. 2011;21(10):1616-25. doi: 10.1101/gr.122705.111. PMID: 21880779.

15. Moreno-Gallego JL, Chou SP, Di Rienzi SC, Goodrich JK, Spector TD, Bell JT, Youngblut ND, Hewson I, Reyes A, Ley RE. Virome Diversity Correlates with Intestinal Microbiome Diversity in Adult Monozygotic Twins. Cell Host Microbe. 2019;25(2):261272.e5. doi: 10.1016/j.chom.2019.01.019. PMID: 30763537.

16. Shkoporov AN, Clooney AG, Sutton TDS, Ryan FJ, Daly KM, Nolan JA, McDonnell SA, Khokhlova EV, Draper LA, Forde A, Guerin E, Velayudhan V, Ross RP, Hill C. The 

microbial transplantation. Microbiome. 2018;6(1):220. doi: 10.1186/s40168-018-0598-x. PMID: 30526683. Filtrate Transfer for Treating Patients With Clostridium difficile Infection. Gastroenterology. 2017;152(4):799-811.e7. doi: 10.1053/j.gastro.2016.11.010. PMID: 27866880.

19. Pulipati P, Sarkar P, Jakkampudi A, Kaila V, Sarkar S, Unnisa M, Reddy DN, Khan M, Talukdar R. The Indian gut microbiota-Is it unique? Indian J Gastroenterol. 2020;39(2):133-140. doi: 10.1007/s12664-020-01037-8. PMID: 32388710. Allin KH, Hansen T, Arumugam M, Vestergaard H, Pedersen O, Pereira V, Abraham P, Tripathi R, Wadhwa N, Bhatnagar S, Prakash VG, Radha V, Anjana RM, Mohan V, Takeda K, Kurakawa T, Nair GB, Das B. An Improved Method for High Quality Metagenomics DNA Extraction from Human and Environmental Samples. Sci Rep. 2016;6:26775. doi: 10.1038/srep26775. PMID: 27240745.

21. Zolfo M, Pinto F, Asnicar F, Manghi P, Tett A, Bushman FD, Segata N. Detecting contamination in viromes using ViromeQC. Nat Biotechnol. 2019;37(12):1408-1412. doi: 10.1038/s41587-019-0334-5. PMID: 31748692. 

assesses the quality and completeness of metagenome-assembled viral genomes. Nat Biotechnol. 2021;39(5):578-585. doi: 10.1038/s41587-020-00774-7. PMID: 33349699. Mucosal-Luminal Interface. Front Cell Infect Microbiol. 2020;10:582187. doi: 10.3389/fcimb.2020.582187. PMID: 33194818. Bacteriophages, the Most Abundant Virus in the Human Gut. Cell Host Microbe. 2018;24(5):653-664.e6. doi: 10.1016/j.chom.2018.10.002. PMID: 30449316. Discovery of an expansive bacteriophage family that includes the most abundant viruses from the human gut. Nat Microbiol. 2018;3(1):38-46. doi: 10.1038/s41564-017-0053-y. PMID: 29133882. Richardson LJ, Salazar GA, Smart A, Sonnhammer ELL, Hirsh L, Paladin L, Piovesan D, Tosatto SCE, Finn RD. The Pfam protein families database in 2019. Nucleic Acids Res. 2019;47(D1):D427-D432. doi: 10.1093/nar/gky995. PMID: 30357350. 
28. Guerin E, Shkoporov AN, Stockdale SR, Comas JC, Khokhlova EV, Clooney AG, Daly KM, Draper LA, Stephens N, Scholz D, Ross RP, Hill C. Isolation and characterisation of ФcrAss002, a crAss-like phage from the human gut that infects Bacteroides xylanisolvens. Microbiome. 2021;9(1):89. doi: 10.1186/s40168-021-01036-7. PMID: 33845877. calculating average nucleotide identity. Int J Syst Evol Microbiol. 2016;66(2):1100-1103. doi: 10.1099/ijsem.0.000760. PMID: 26585518. Endocrinol (Lausanne). 2019;10:784. doi: 10.3389/fendo.2019.00784. PMID: 31849833. 10.1093/ve/veab022. PMID: 33959381.

32. Yolken RH, Jones-Brando L, Dunigan DD, Kannan G, Dickerson F, Severance E, of the human oropharyngeal virome and is associated with changes in cognitive functions in humans and mice. Proc Natl Acad Sci U S A. 2014;111(45):16106-11. doi: 10.1073/pnas.1418895111. PMID: 25349393.

33. Edwards RA, Vega AA, Norman HM, Ohaeri M, Levi K, Dinsdale EA, Cinek O, Aziz RK, McNair K, Barr JJ, Bibby K, Brouns SJJ, Cazares A, de Jonge PA, Desnues C, Díaz 
Muñoz SL, Fineran PC, Kurilshikov A, Lavigne R, Mazankova K, McCarthy DT,

Nobrega FL, Reyes Muñoz A, Tapia G, Trefault N, Tyakht AV, Vinuesa P, Wagemans J, evolution of the widespread human gut virus crAssphage. Nat Microbiol. 2019;4(10):1727-1736. doi: 10.1038/s41564-019-0494-6. PMID: 31285584. human gut phageome. Proc Natl Acad Sci U S A. 2016;113(37):10400-5. doi: 10.1073/pnas.1601060113. PMID: 27573828. human gut virome. Proc Natl Acad Sci U S A. 2013;110(30):12450-5. doi: 


\section{$615 \quad$ Figure Legends}

616 Figure 1. Data summary and quality check. (A) The number of reads before (black bars) and

617 after (grey bars) quality control is shown. Subject identity and the type of samples are indicated

618 on the x-axis. The VLP DNA represents both dsDNA and ssDNA obtained from purified VLPs.

619 Total microbial DNA represents dsDNA. (B) Quality filtered reads were analyzed with

620 ViromeQC tool. The y-axis represents the virus enrichment scores and the corresponding

621 samples are indicated on the x-axis. (C) Functional profiles of the contigs obtained from the

622 VLP-DNA and the total microbial DNA. Protein features were predicted and annotated by the

623 MG-RAST pipeline. The abundance of the top most-abundant 12 categories in the Subsystems

624 database is plotted for the samples identified on the x-axis. The identified classes are listed at the

625 bottom of the graph and their proportion is indicated on the y-axis. (D) Mining tools were used to

626 identify viral contigs after de-novo assembly and the redundancy was removed within each

627 sample. The CheckV tool was used to analyze the quality of the assembled genomes. The

628 proportion of viral contigs classified as "complete genomes", "high-quality genomes", "medium-

629 quality genomes", "low-quality" and "not-determined" in each sample are depicted. Subject

630 identity is indicated on the x-axis. VLP-derived contigs are depicted as "V" and the total

631 microbial DNA-derived viral contigs are depicted as "vM".

632

633 Figure 2. Taxonomic identification of viral sequences. (A) The high-quality reads mapped to

634 the viral RefSeq database are depicted by a doughnut chart. Each of the viral categories is shown

635 by a different color. Each ring shows the distribution of various viruses in a sample. The

636 sequence of samples starting with the innermost ring and going outwards is F3, F4, F5, F7, F11,

637 F12, F13, F14, F16, F18, F21 and F29. (B) Taxonomic assignment by the Kaiju program. 
638 Contigs that were identified as viral by the virus mining tools were assigned taxonomy based on 639 protein alignment using Viral RefSeq databases, 1.1, 2.1, and 3.1. The pie chart is showing the

640 identified viral families. (C) Taxonomic identification of the viral contigs based on the

641 assignment of the vCONTACT2-generated clusters using Demovir and the TrEMBL database.

642 The pie chart is showing the proportion of the identified viral families (D) Taxonomic

643 identification of the viral contigs based on the assignment of the vCONTACT2-generated

644 clusters using the ProkaryoticViralRefSeq94-Merged database. The pie chart is showing the

645 proportion of the identified viral families. (E) The pie chart showing the proportion of each of

646 the ten crAss-like phage genera and unclassified crAss-like phages.

Figure 3. Identification of shared viral families. A doughnut chart showing viral families, that are shared across all samples. Each circle represents a sample. The sequence of samples starting with the innermost ring and going outwards is F3, F4, F5, F7, F11, F12, F13, F14, F16, F18, F21 and F29. (A) Viral families were identified by the protein-alignment-based method using the

652 program Kaiju. Families that are present in all samples are shown. (B) Protein-based clustering

653 of the viral contigs was performed by vConTACT2. Clusters were annotated using the TrEMBL

654 database. Families shared by all samples are listed. (C) Clusters generated by vCONTACT2

655 were annotated using the ProkaryoticViralRefSeq94-Merged database. Families shared by all 656 samples are listed.

Figure 4. Diversities of the viral and bacterial populations and correlation analyses. (A)

659 Species richness was determined with the Vegan package of R. Chao 1 indices are depicted on 660 the $y$-axis with respective samples on the $\mathrm{x}$-axis. The richness of bacterial species, phages that 
661 are associated with the host and the free virus (VLP) are represented with black bars, gray bars

662 and white bars, respectively. (B) Species richness correlations of bacterial species richness with

663 free viral species (solid line and filled circles; coefficient of correlation ( $\mathrm{r}$ ) is -0.12 ) as well as

664 with phages associated with hosts (dotted line and open circles; coefficient of correlation (r) is -

665 0.60) are shown.

666

667 Figure 5. Bacteriophage lifestyle. Viral contigs were clustered as vOTUs with ClusterGenomes

668 and ORFs were predicted in each vOTU, using Prodigal. ORFs were annotated using a custom

669 set of HMM profiles of lysogeny-associated genes. (A) Black bars show the total number of

670 vOTUs (y-axis) in each sample (x-axis) and the grey bars show the number of vOTUs in which

671 lysogeny-associated genes were detected. The calculated percentage of lysogenic vOTUs is

672 indicated on top of the bars. (B) The origin of the identified lysogenic vOTUs is shown.

673

674 Supplemental Information

675 Document Supl_Tables

676

677 Acknowledgments

678 Dr. Bhabatosh Das, Translational Health Science and Technology Institute is gratefully

679 acknowledged for his valuable suggestions on the manuscript.

\section{Data availability statement}

682 https://dataview.ncbi.nlm.nih.gov/object/PRJNA792685?reviewer=tedv6alaa2mvci3sliad55k7ok 


\section{Author contributions}

685 KB: Conception; design; acquisition, analysis and interpretation of data

686 HS: Data collection and final approval

687 AG, ADP, MK: Data analysis, revisiting the manuscript and final approval

688 SV: Conception, revisiting the manuscript and final approval

689 Funding

690 This work was supported by the Department of Biotechnology, Government of India grant no.

691 BT/PR18657/BIC/101/507/2016 to KB. MK acknowledges the grant nos. VIR(25)/2019/ECD-1, and

692 ISRM/12(33)/2019 by the Indian Council of Medical Research, and grant no. EMR/2017/002299 by the

693 Scientific and Engineering Research Board.

\section{Disclosures}

695 The authors declare no competing interests.

696

697

698 
Figure 1A

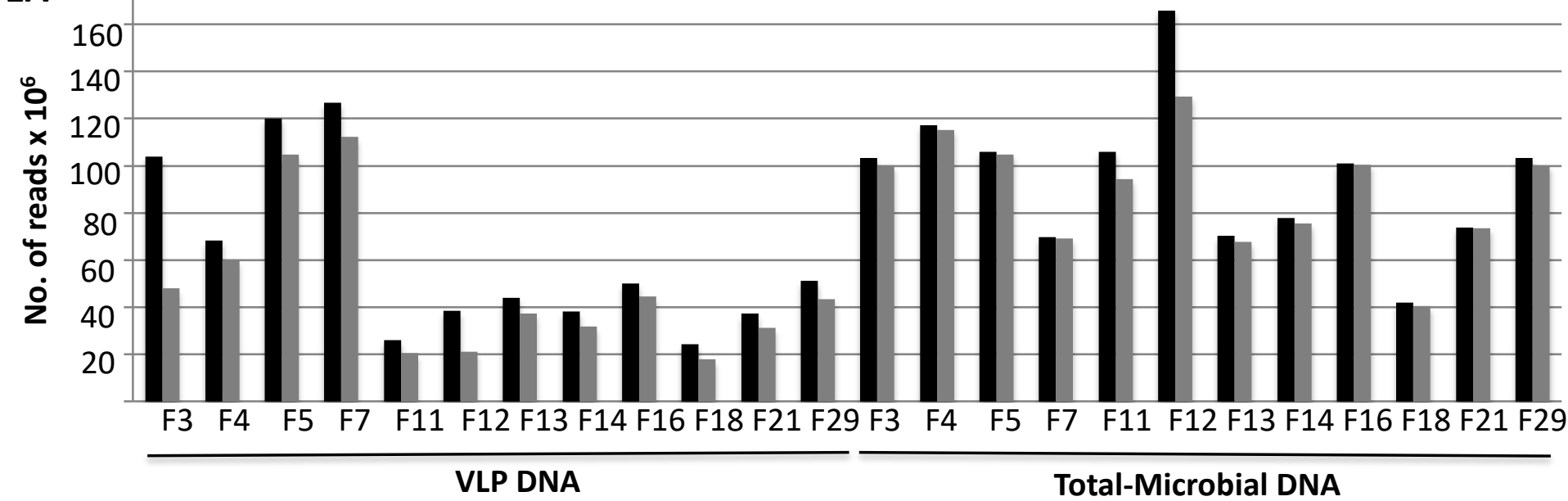

Figure 1B

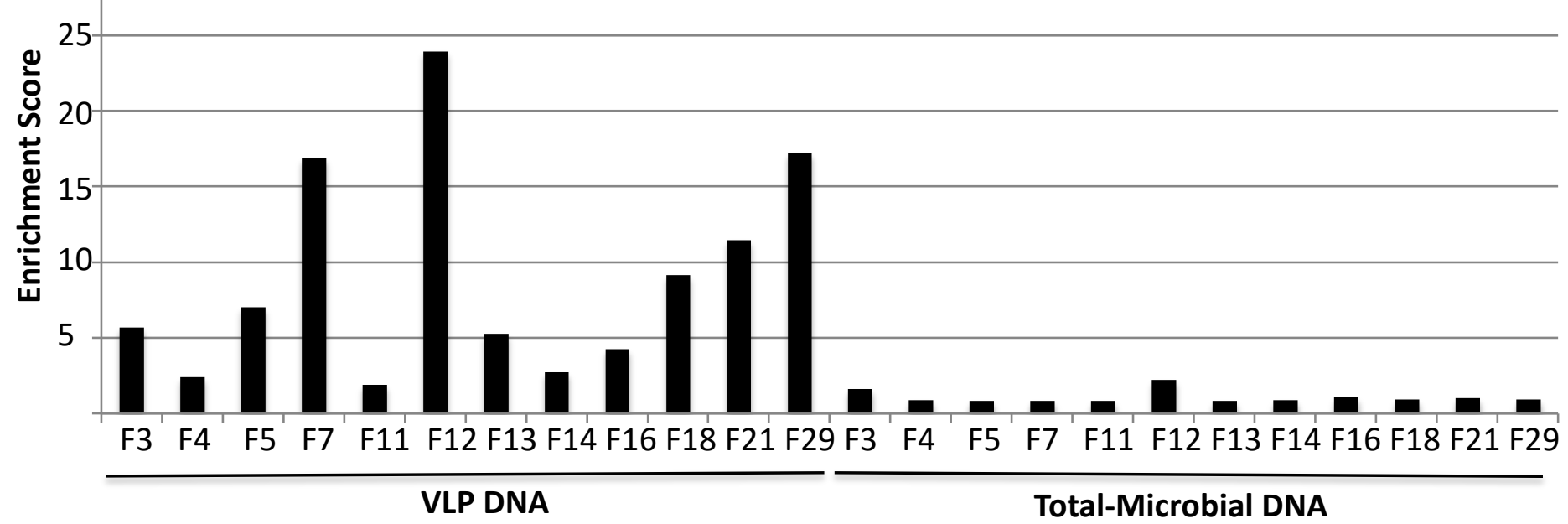




\section{Figure 1C}

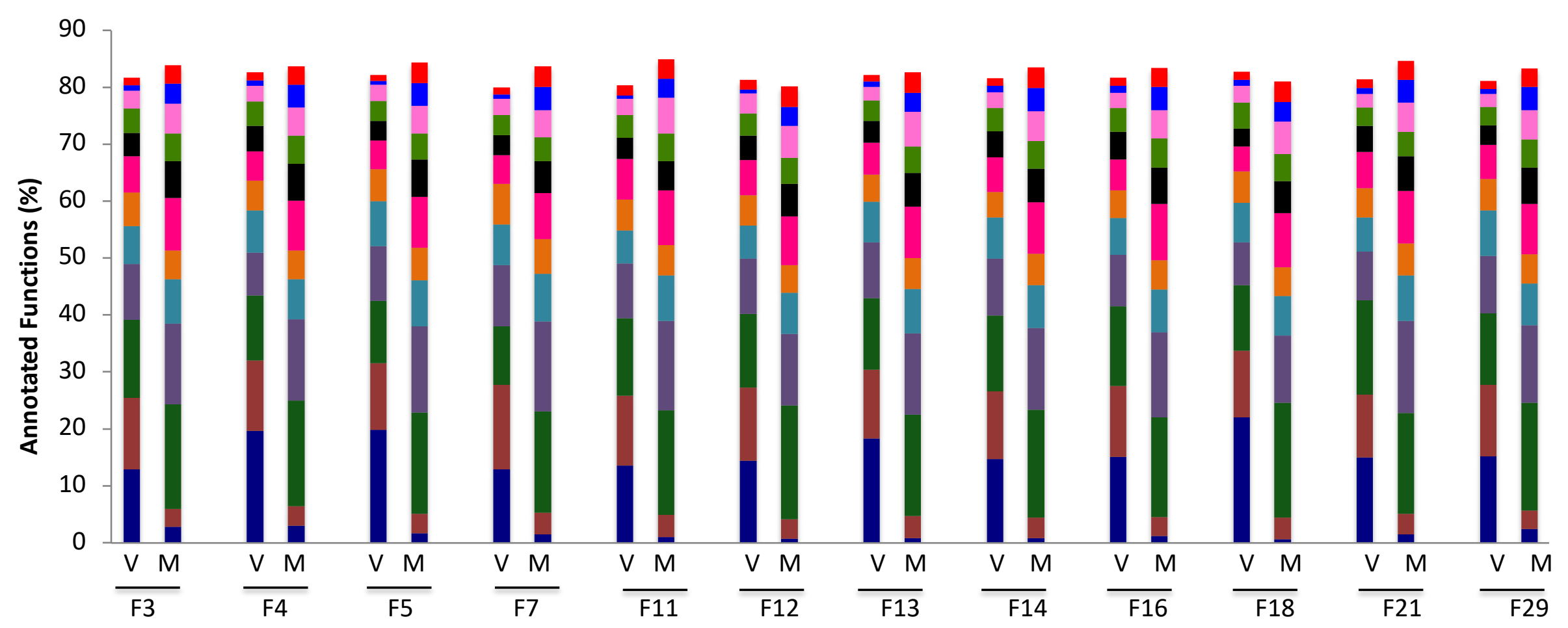

- Phages, Prophages, Transposable elements, Plasmids

- Carbohydrates

- DNA Metabolism

- Amino Acids and Derivatives

- Cofactors, Vitamins, Prosthetic Groups, Pigments

- Respiration n Clustering-based subsystems

- Protein Metabolism

- RNA Metabolism

- Cell Wall and Capsule

nucleosides and Nucleotides

- Stress Response 
Figure 1D

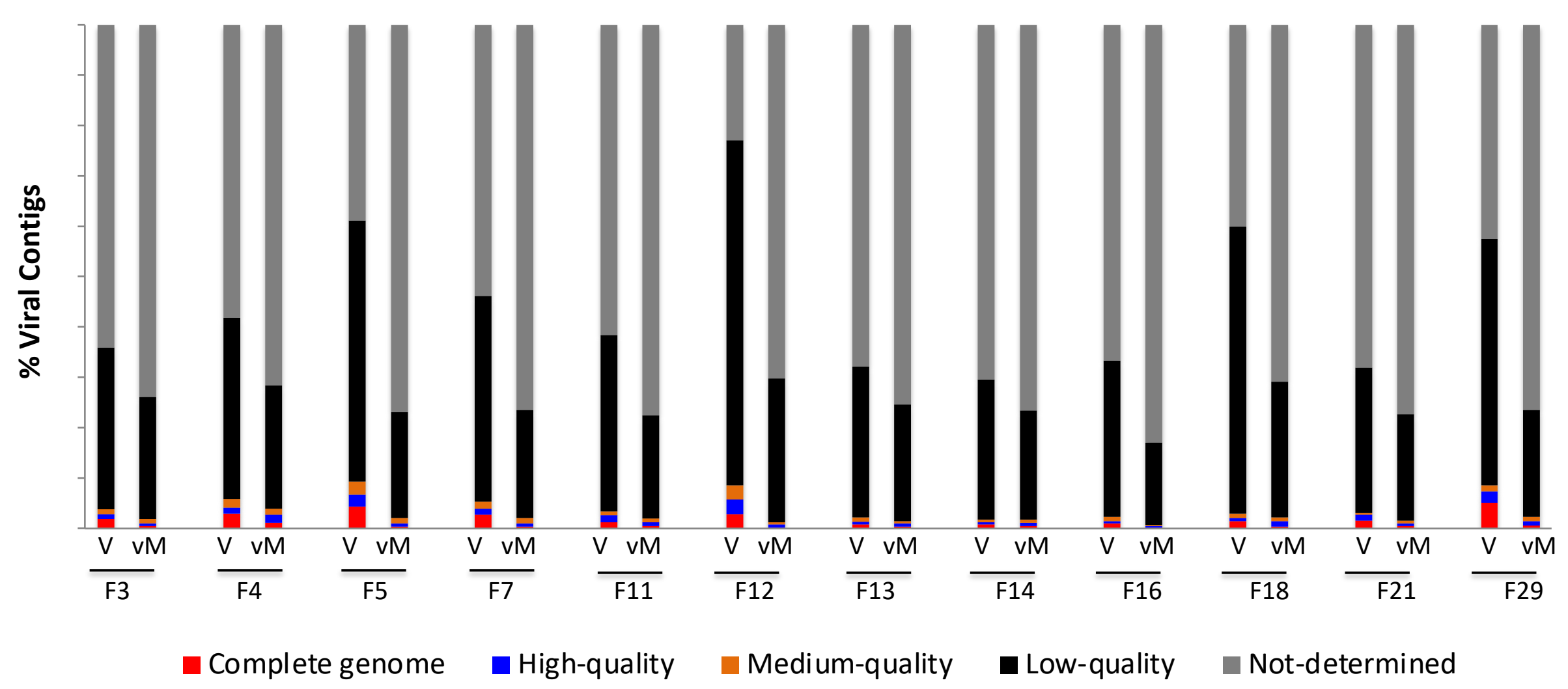


Figure 2A

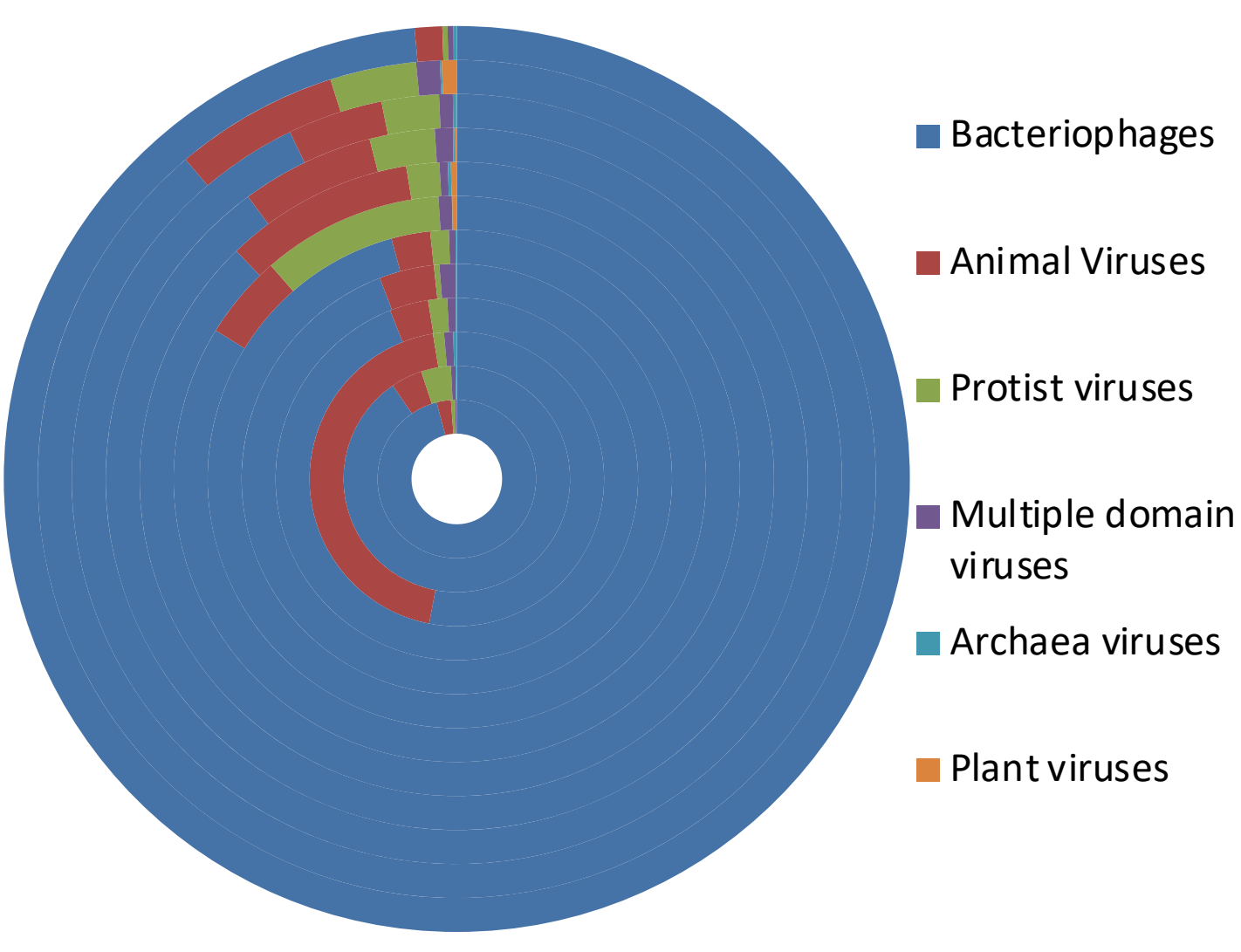




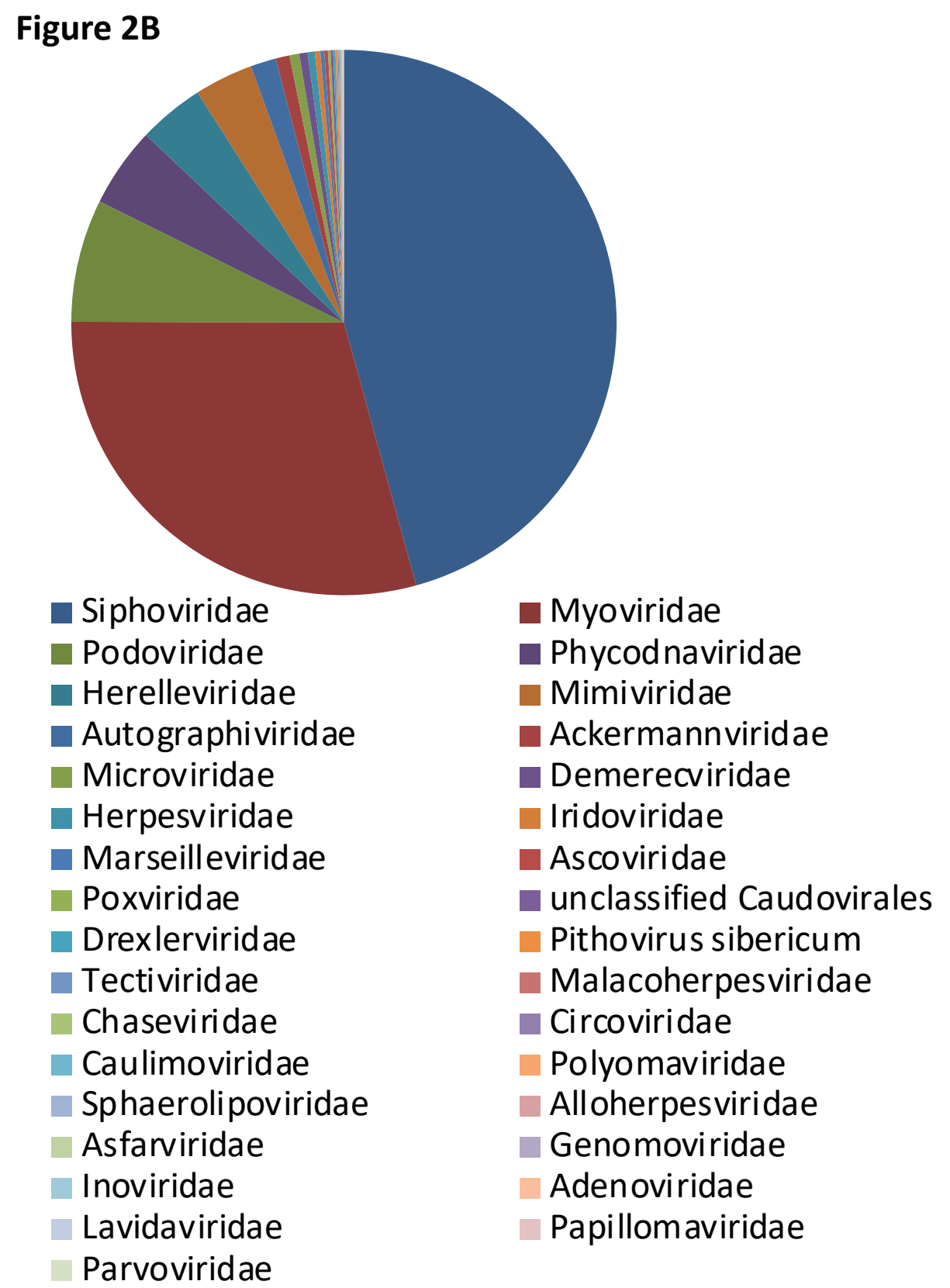




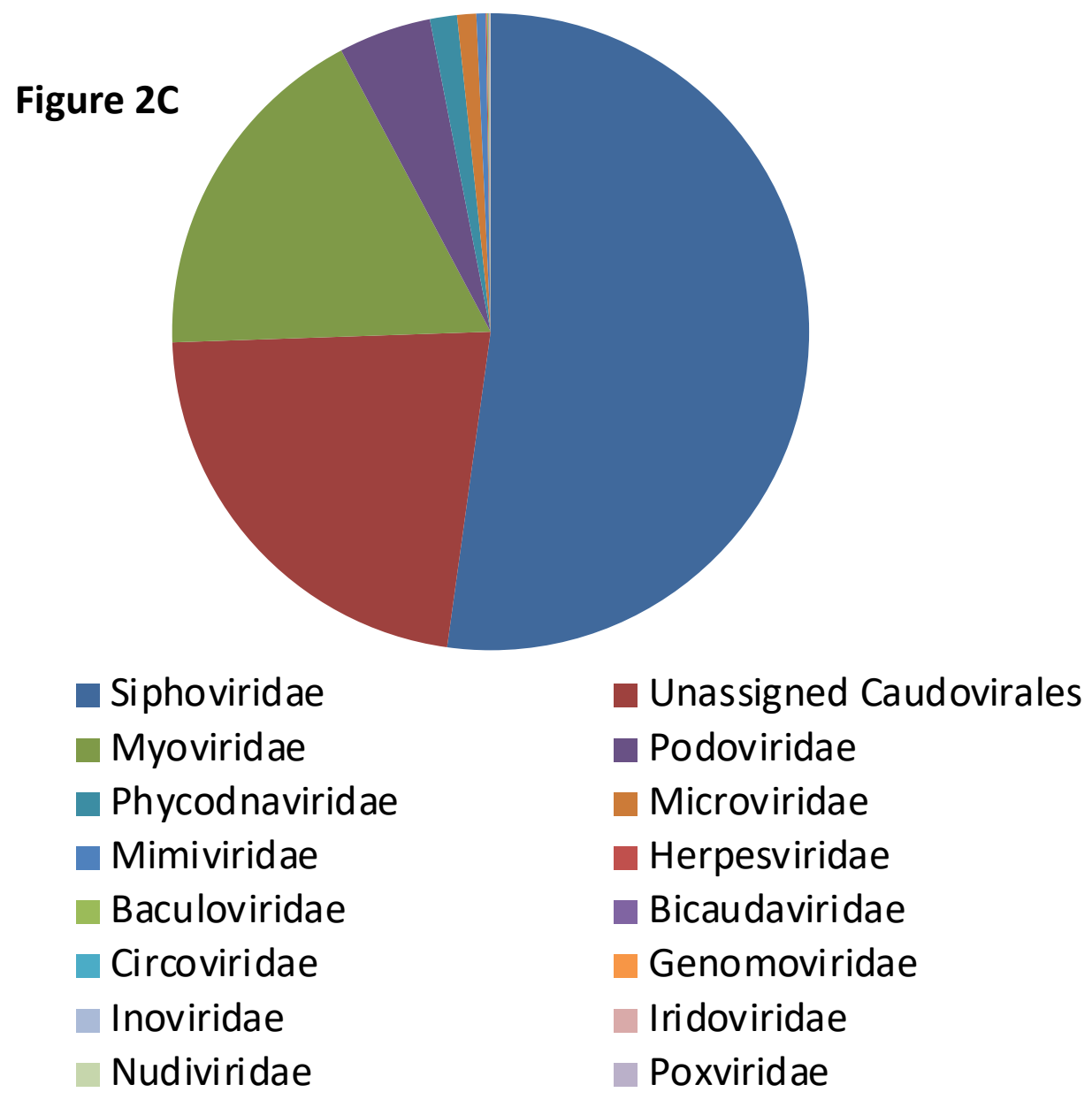




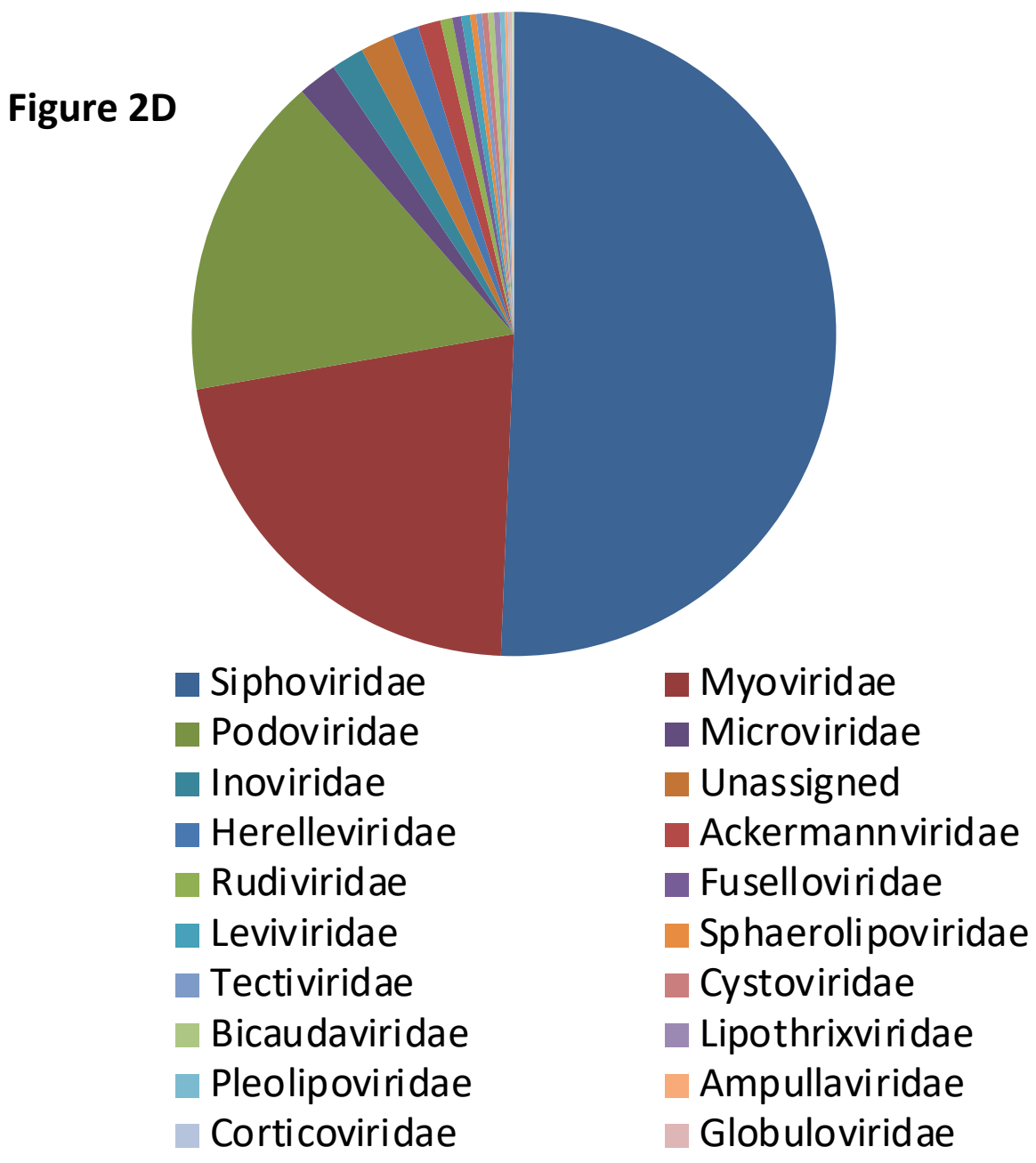


Figure 2E

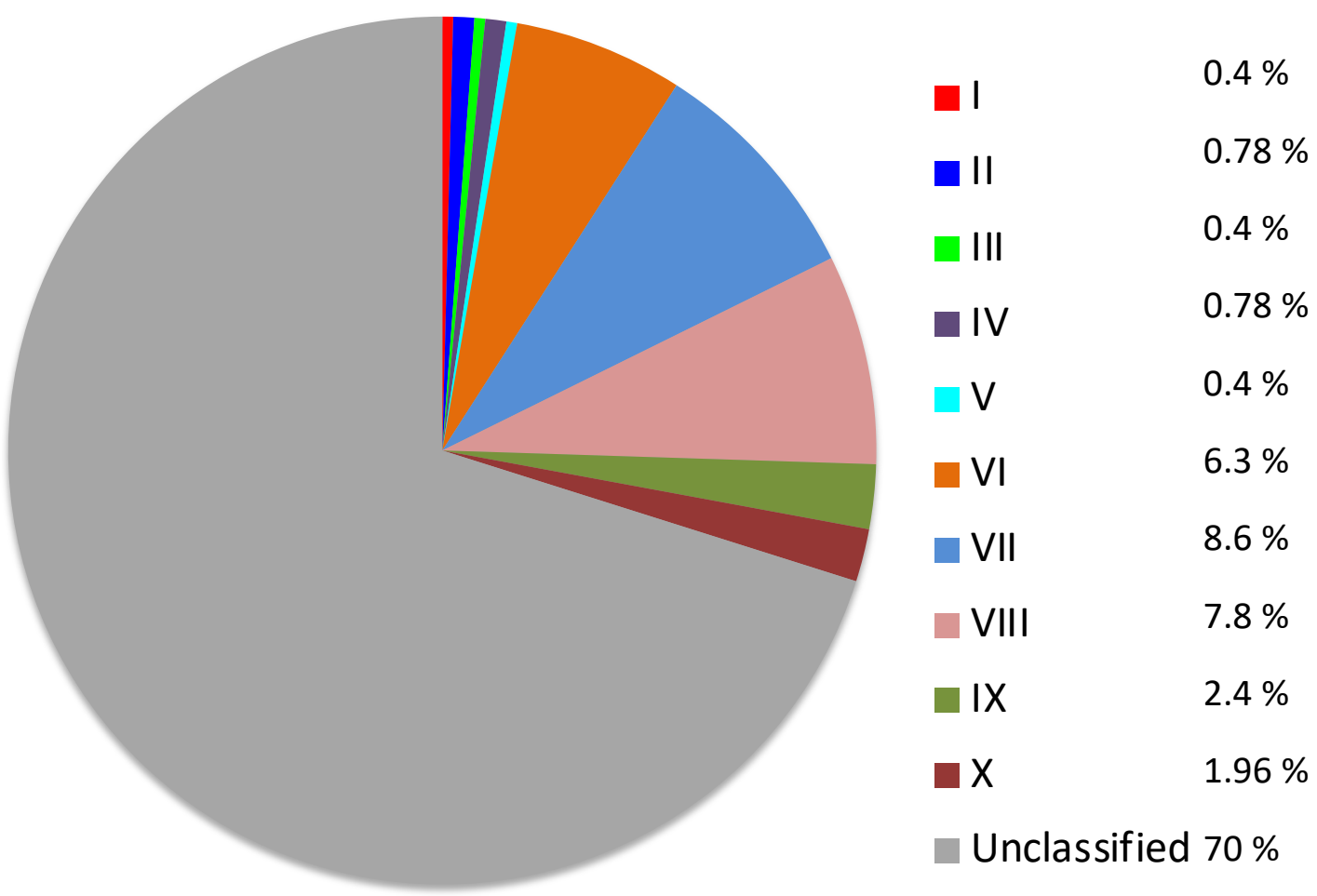


Figure 3A

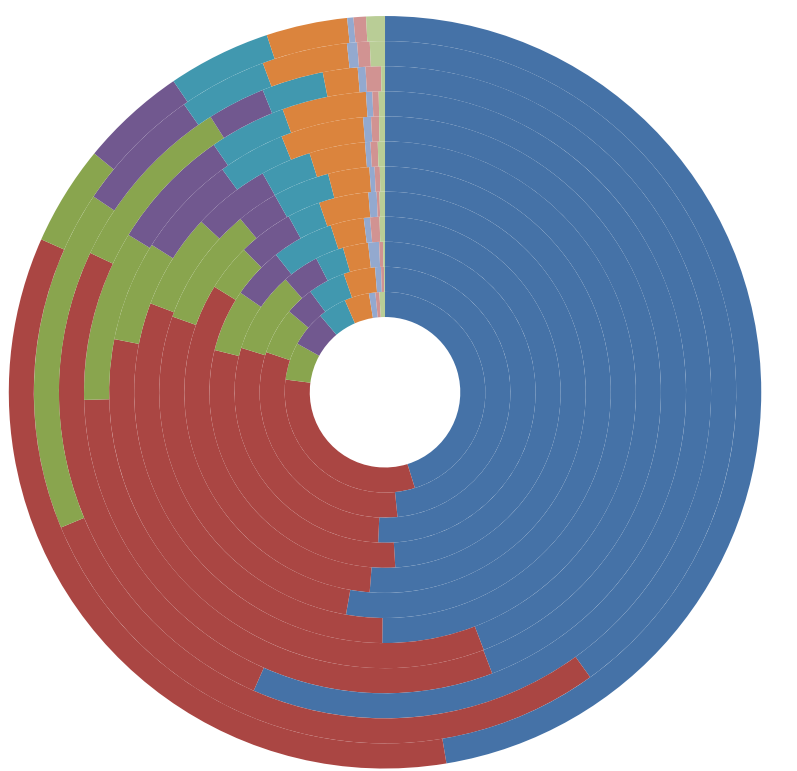
- Siphoviridae
Myoviridae
- Podoviridae
- Phycodnaviridae
- Herelleviridae
- Mimiviridae
Microviridae
- Demerecviridae
Herpesviridae 


\section{Figure 3B}

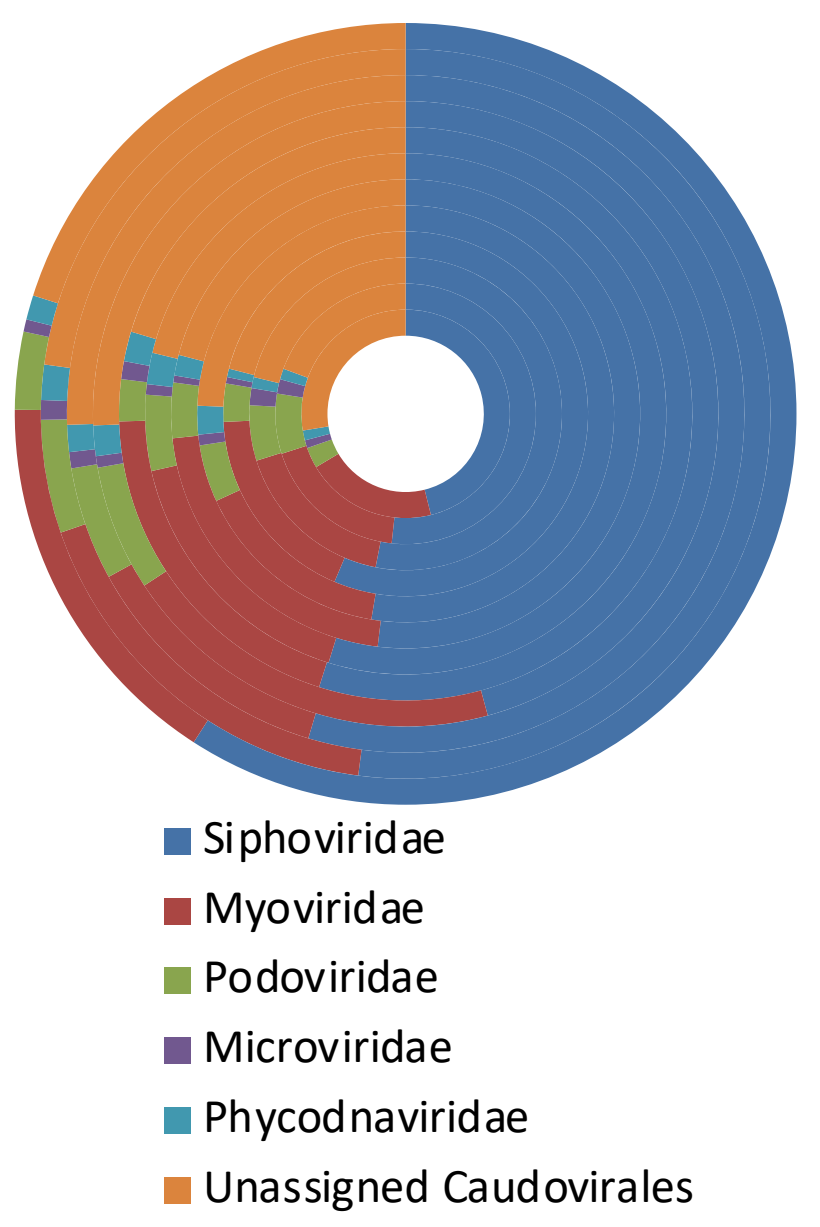




\section{Figure 3C}

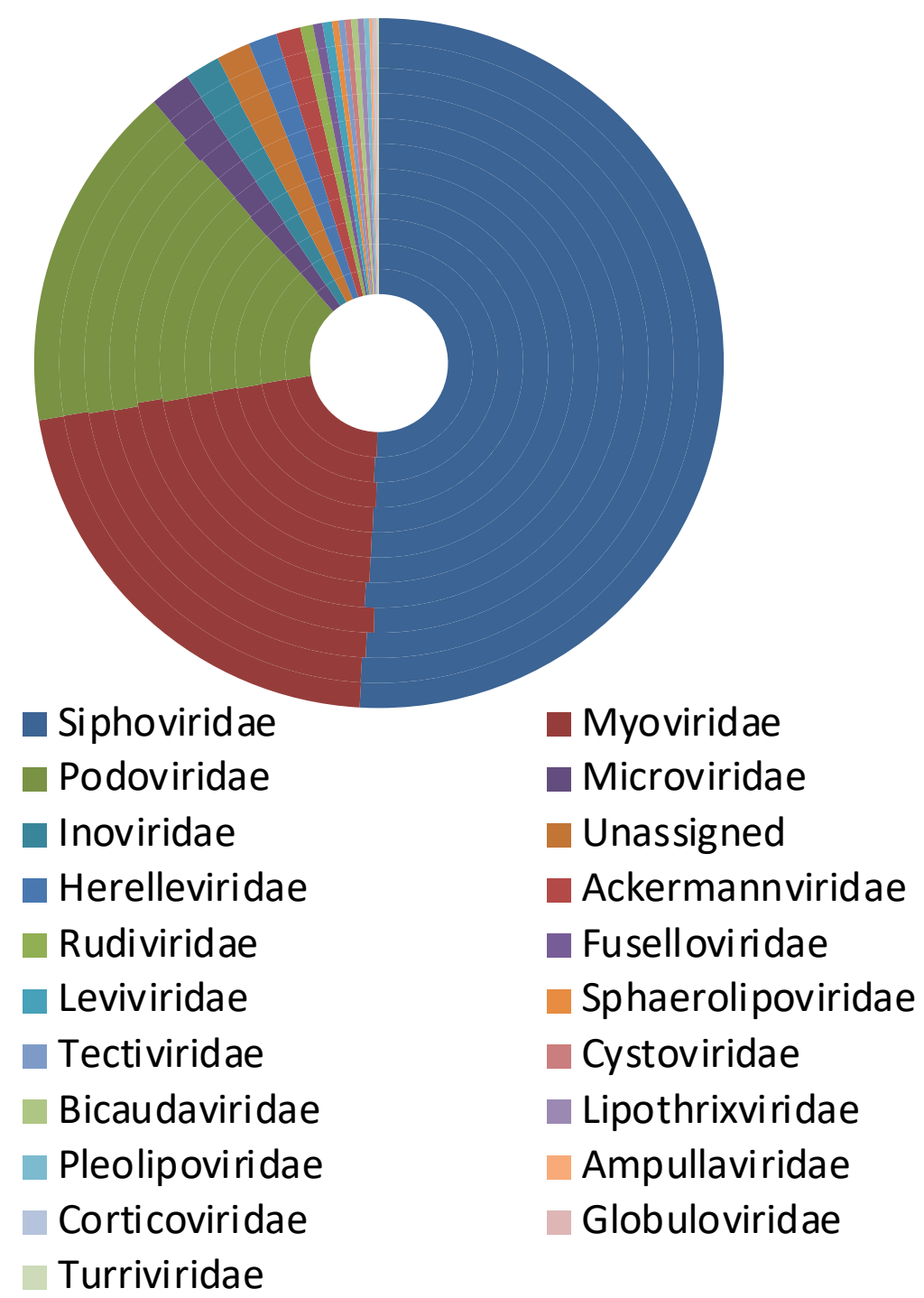




\section{Figure 4A}

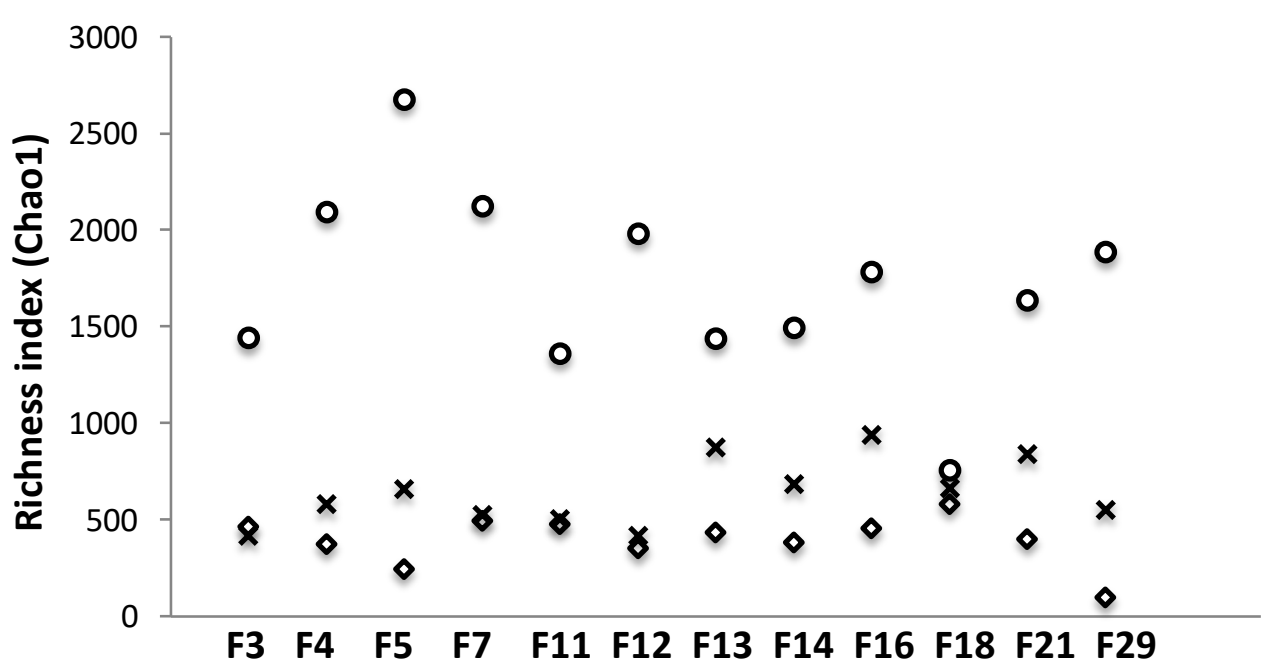

Figure 4B

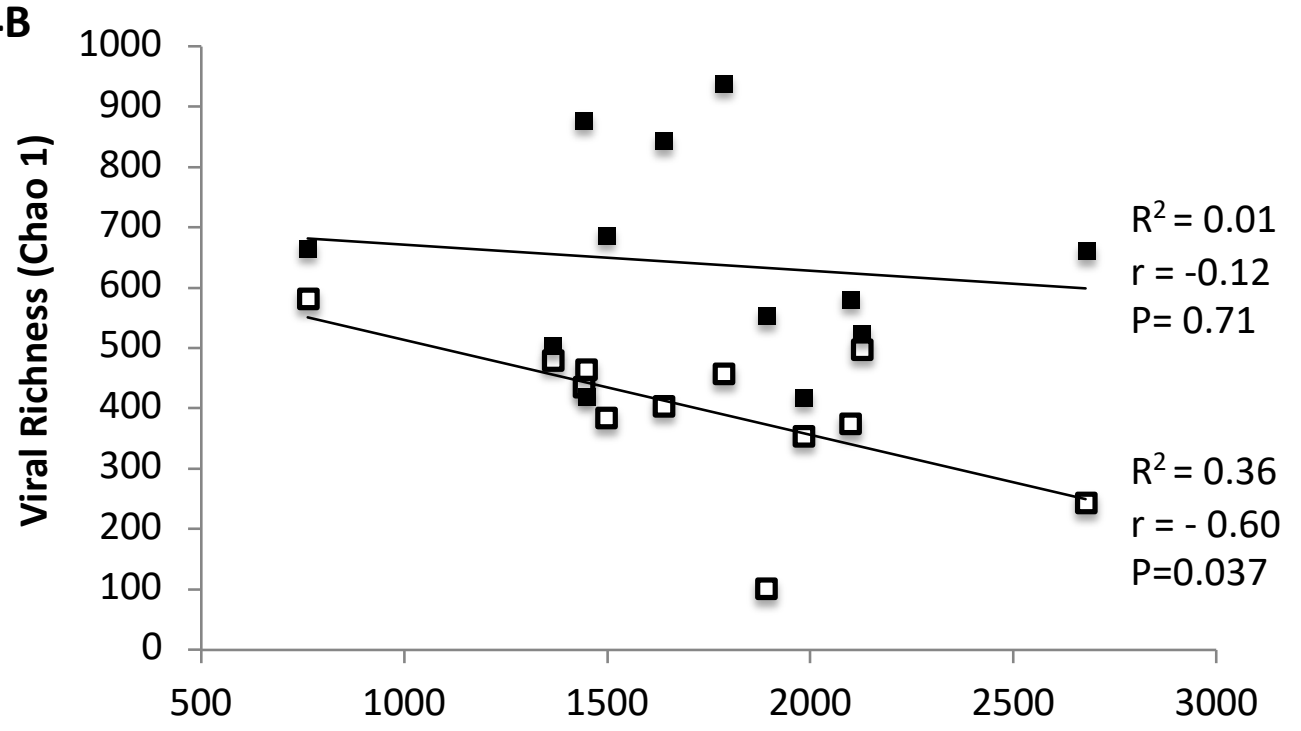

Bacterial Richness (Chao 1) 
Figure 5A

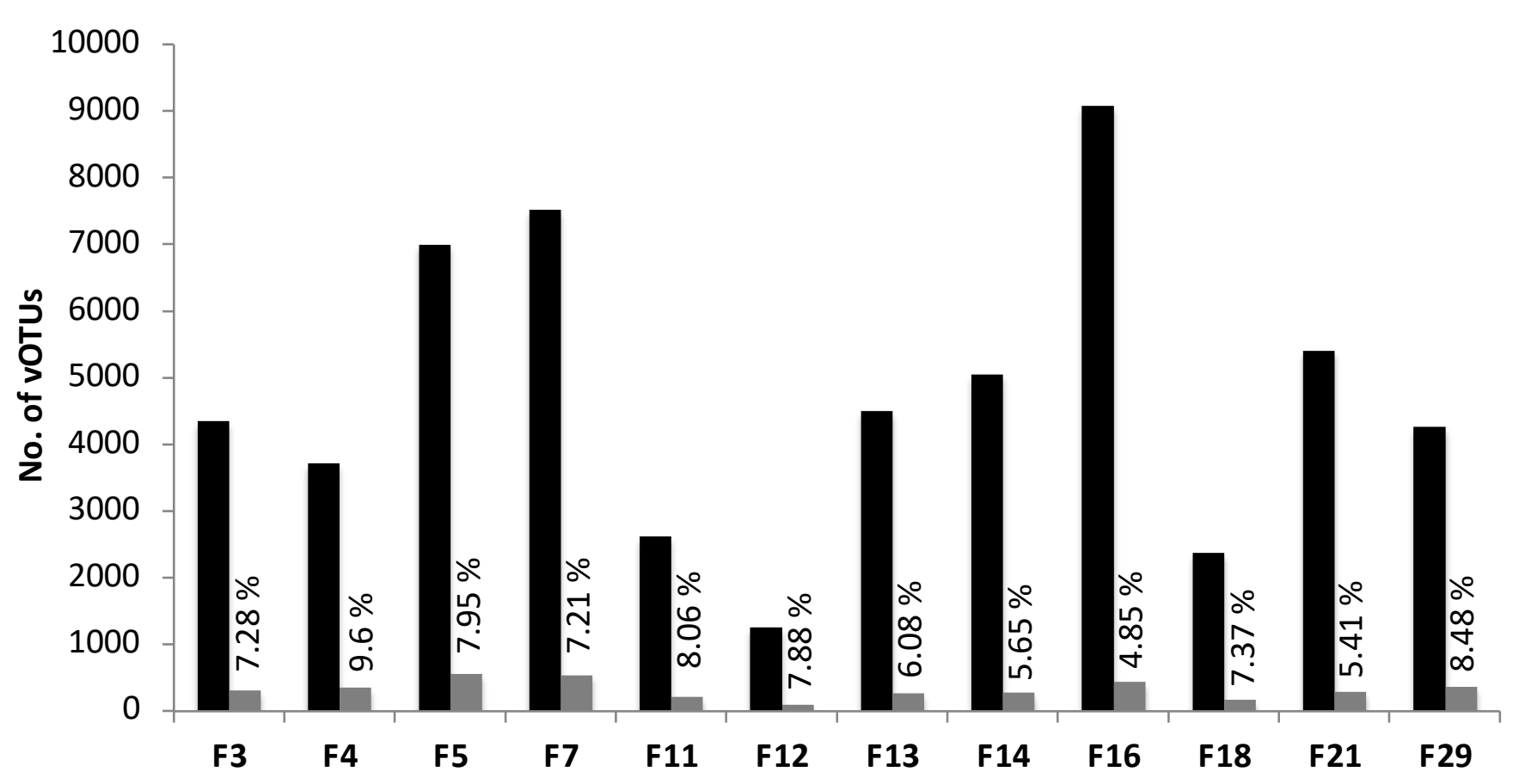

Figure 5B

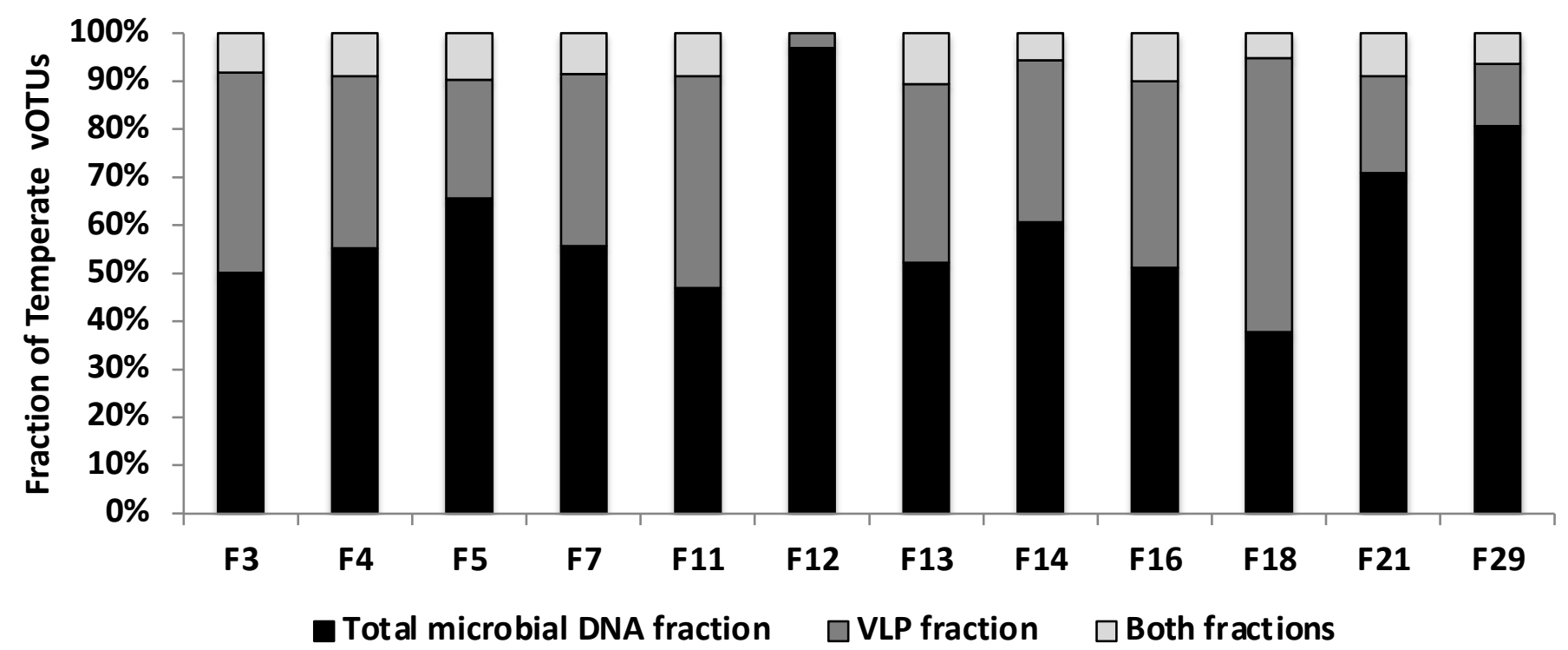


Table S1. Read Summary

\begin{tabular}{|c|c|c|c|c|}
\hline \multirow{2}{*}{ Sample i.d. } & \multicolumn{2}{|c|}{ Number of VLP DNA-derived reads } & \multicolumn{2}{c|}{ Number of total-microbial DNA-derived reads } \\
\cline { 2 - 5 } & Total & Mapped to viral RefSeq & Total & Mapped to viral RefSeq \\
\hline F3 & 55945748 & $27066(0.05 \%)$ & 103310208 & $27083(0.03 \%)$ \\
\hline F4 & 68388278 & $60763(0.09 \%)$ & 117258248 & $21582(0.02 \%)$ \\
\hline F5 & 120277474 & $394972(0.3 \%)$ & 105878448 & $6571(0.006 \%)$ \\
\hline F7 & 126813688 & $39176(0.03 \%)$ & 69697178 & $34207(0.05 \%)$ \\
\hline F11 & 26006146 & $38904(0.15 \%)$ & 106044330 & $66301(0.06 \%)$ \\
\hline F12 & 38375058 & $81769(0.211 \%)$ & 165786894 & $169968(0.1 \%)$ \\
\hline F13 & 44020188 & $68081(0.15 \%)$ & 70267208 & $54502(0.08 \%)$ \\
\hline F14 & 38132234 & $26052(0.07 \%)$ & 77844958 & $46839(0.06 \%)$ \\
\hline F16 & 50049542 & $41176(0.08 \%)$ & 100960596 & $36169(0.04 \%)$ \\
\hline F18 & 24414020 & $38032(0.16 \%)$ & 42099008 & $29505(0.07 \%)$ \\
\hline F21 & 37447036 & $53276(0.14 \%)$ & 73885912 & $34413(0.05 \%)$ \\
\hline F29 & 51299028 & $568553(1.11 \%)$ & 103310208 & $77819(0.07 \%)$ \\
\hline Total & $\mathbf{6 8 1 1 6 8 4 4 0}$ & $\mathbf{1 4 3 7 8 2 0}$ & $\mathbf{1 1 3 6 3 4 3 1 9 6}$ & $\mathbf{6 0 4 9 5 9}$ \\
\hline
\end{tabular}


Table S2. Summary of metagenome assembly

\begin{tabular}{|c|c|c|c|c|c|c|c|c|}
\hline \multirow[t]{2}{*}{ Sample i.d. } & \multicolumn{2}{|c|}{ No. of reads } & \multicolumn{2}{|c|}{$\begin{array}{l}\text { No. of total contigs } \\
\text { (MEGAHIT) }\end{array}$} & \multicolumn{2}{|c|}{$\begin{array}{l}\text { No. of contigs } \\
\text { (> } 1000 \mathrm{bp})\end{array}$} & \multicolumn{2}{|c|}{ Largest Contig (bp) } \\
\hline & VLP-DNA & $\begin{array}{c}\text { Total } \\
\text { Microbial- } \\
\text { DNA }\end{array}$ & VLP-DNA & $\begin{array}{l}\text { Total } \\
\text { Microbia } \\
\text { I-DNA }\end{array}$ & $\begin{array}{l}\text { VLP- } \\
\text { DNA }\end{array}$ & $\begin{array}{c}\text { Total } \\
\text { Microbi } \\
\text { al-DNA }\end{array}$ & VLP-DNA & $\begin{array}{c}\text { Total } \\
\text { Microbial- } \\
\text { DNA }\end{array}$ \\
\hline F3 & 55945748 & 103310208 & 77253 & 179130 & 11055 & 41248 & 737163 & 725802 \\
\hline F4 & 68388278 & 117258248 & 48221 & 142785 & 10225 & 38742 & 796189 & 796189 \\
\hline F5 & 120277474 & 105878448 & 55249 & 454755 & 7655 & 88264 & 640931 & 411643 \\
\hline F7 & 126813688 & 69697178 & 56594 & 363684 & 7594 & 86724 & 405150 & 330991 \\
\hline F11 & 26006146 & 106044330 & 15115 & 98940 & 2916 & 24738 & 265523 & 368663 \\
\hline F12 & 38375058 & 165786894 & 29423 & 109813 & 306 & 21751 & 31161 & 232869 \\
\hline F13 & 44020188 & 70267208 & 58699 & 174692 & 11561 & 35666 & 176112 & 814305 \\
\hline F14 & 38132234 & 77844958 & 50041 & 201298 & 7565 & 45857 & 294552 & 1104472 \\
\hline F16 & 50049542 & 100960596 & 95950 & 360992 & 15590 & 69923 & 535552 & 534186 \\
\hline F18 & 24414020 & 42099008 & 38350 & 76588 & 5207 & 18440 & 154147 & 537610 \\
\hline F21 & 37447036 & 73885912 & 34304 & 229747 & 6793 & 55788 & 250352 & 667724 \\
\hline F29 & 51299028 & 103310208 & 302306 & 259094 & 7145 & 63798 & 489932 & 633385 \\
\hline Total & 681168440 & 1239722496 & 861505 & 2651518 & 93612 & 590939 & & \\
\hline
\end{tabular}


Table S3. Summary of data mining to recover viral sequences

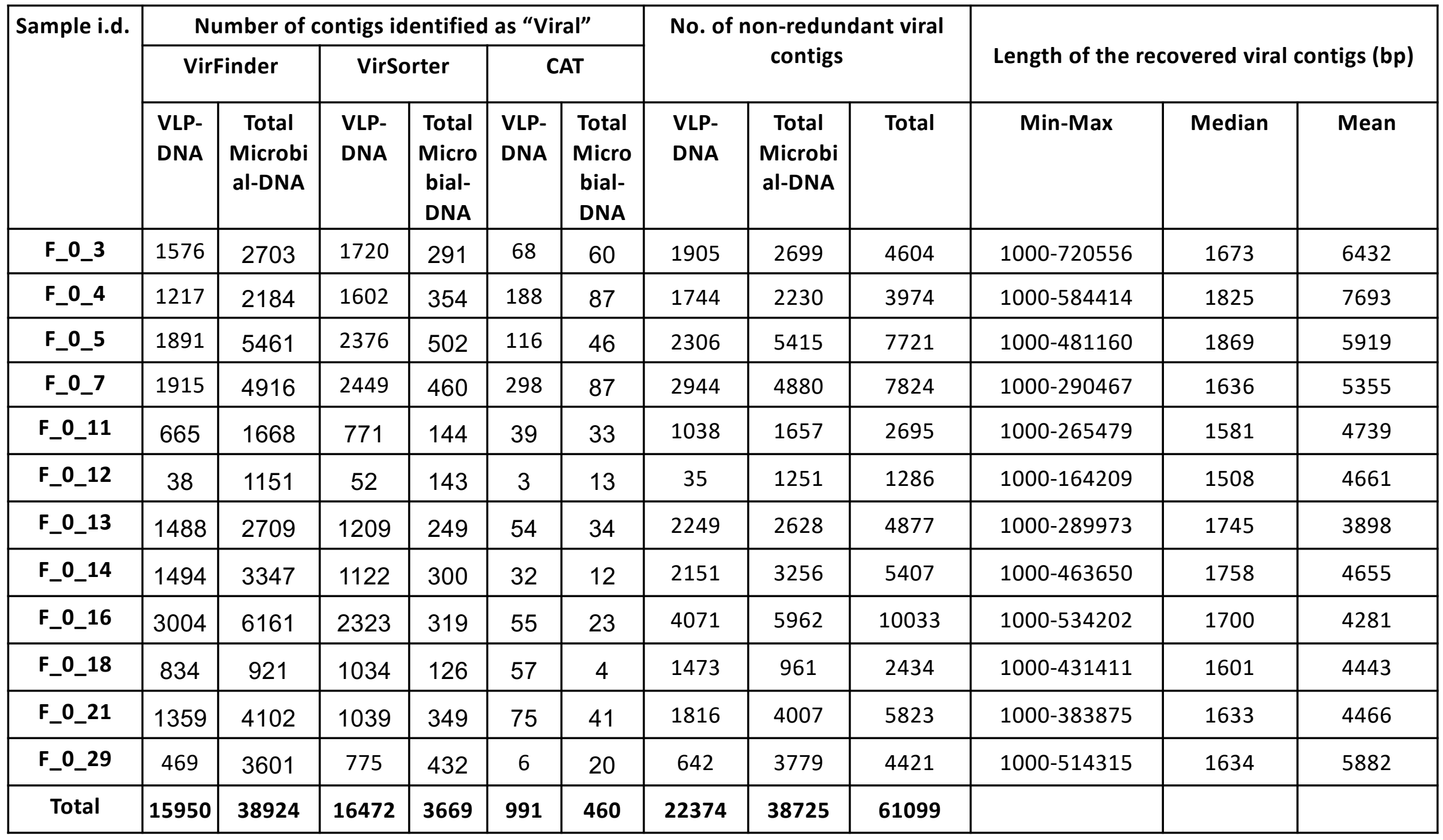


Table S4. Summary of CheckV output

\begin{tabular}{|c|c|c|c|c|c|c|c|c|c|c|c|c|c|c|c|}
\hline \multirow[t]{2}{*}{$\begin{array}{l}\text { Sample } \\
\text { i.d. }\end{array}$} & \multicolumn{3}{|c|}{$\begin{array}{l}\text { No. of "complete" } \\
\text { genomes }\end{array}$} & \multicolumn{3}{|c|}{$\begin{array}{l}\text { No. of "high quality" } \\
\text { genomes (> } 90 \% \\
\text { complete genomes) }\end{array}$} & \multicolumn{3}{|c|}{$\begin{array}{c}\text { No. of "Undetermined } \\
\text { quality" }\end{array}$} & \multicolumn{3}{|c|}{$\begin{array}{l}\text { No. of "medium } \\
\text { quality" } \\
\text { (> 50-90\% complete } \\
\text { genomes) }\end{array}$} & \multicolumn{3}{|c|}{$\begin{array}{c}\text { No. of "low quality" } \\
\text { (>0-50\% complete } \\
\text { genomes) }\end{array}$} \\
\hline & $\begin{array}{l}\text { VLP- } \\
\text { DNA }\end{array}$ & $\begin{array}{l}\text { Total } \\
\text { Micr } \\
\text { obial- } \\
\text { DNA }\end{array}$ & Total & $\begin{array}{l}\text { VLP- } \\
\text { DNA }\end{array}$ & $\begin{array}{c}\text { Total } \\
\text { Micr } \\
\text { obial } \\
- \\
\text { DNA }\end{array}$ & Total & $\begin{array}{l}\text { VLP- } \\
\text { DNA }\end{array}$ & $\begin{array}{l}\text { Total } \\
\text { Micro } \\
\text { bial- } \\
\text { DNA }\end{array}$ & Total & $\begin{array}{l}\text { VLP- } \\
\text { DNA }\end{array}$ & $\begin{array}{l}\text { Total } \\
\text { Micr } \\
\text { obial- } \\
\text { DNA }\end{array}$ & Total & $\begin{array}{l}\text { VLP- } \\
\text { DNA }\end{array}$ & $\begin{array}{l}\text { Total } \\
\text { Micr } \\
\text { obial- } \\
\text { DNA }\end{array}$ & Total \\
\hline F_0_3 & 35 & 12 & 47 & 20 & 16 & 36 & 1221 & 1993 & 3214 & 17 & 24 & 41 & 612 & 654 & 1266 \\
\hline F_0_4 & 51 & 25 & 76 & 21 & 37 & 58 & 1015 & 1596 & 2611 & 31 & 25 & 56 & 637 & 547 & 1184 \\
\hline F_0_5 & 101 & 18 & 119 & 53 & 37 & 90 & 895 & 4164 & 5059 & 60 & 56 & 116 & 1197 & 1140 & 2337 \\
\hline F_0_7 & 79 & 20 & 99 & 37 & 30 & 67 & 1585 & 3733 & 5318 & 42 & 51 & 93 & 1201 & 1046 & 2247 \\
\hline F_0_11 & 13 & 7 & 20 & 14 & 13 & 27 & 639 & 1285 & 1924 & 8 & 13 & 21 & 364 & 339 & 703 \\
\hline F_0_12 & 1 & 2 & 3 & 1 & 8 & 9 & 8 & 878 & 886 & 1 & 6 & 7 & 24 & 357 & 381 \\
\hline F_0_13 & 18 & 9 & 27 & 12 & 18 & 30 & 1527 & 1982 & 3509 & 20 & 12 & 32 & 672 & 607 & 1279 \\
\hline F_0_14 & 17 & 15 & 32 & 10 & 21 & 31 & 1516 & 2494 & 4010 & 11 & 23 & 34 & 597 & 703 & 1300 \\
\hline F_0_16 & 40 & 8 & 48 & 19 & 18 & 37 & 2713 & 4944 & 7657 & 33 & 15 & 58 & 1266 & 977 & 2243 \\
\hline F_0_18 & 21 & 3 & 24 & 10 & 11 & 21 & 590 & 681 & 1271 & 13 & 7 & 20 & 839 & 259 & 1098 \\
\hline F_0_21 & 28 & 21 & 49 & 22 & 18 & 40 & 1236 & 3099 & 4335 & 5 & 23 & 28 & 525 & 846 & 1371 \\
\hline F_0_29 & 33 & 23 & 56 & 14 & 30 & 44 & 273 & 2891 & 3164 & 8 & 33 & 41 & 314 & 802 & 1116 \\
\hline Total & 437 & 163 & 600 & 233 & 257 & 490 & 13218 & 29740 & 42958 & 249 & 288 & 547 & 8248 & 8277 & 16525 \\
\hline
\end{tabular}


Table S5. Identification of crAss-like phages

\begin{tabular}{|c|c|c|c|c|c|}
\hline \multirow[t]{2}{*}{ Sample i.d. } & \multirow{2}{*}{$\begin{array}{c}\text { Read } \\
\text { mapping to } \\
\text { crAss-like } \\
\text { phages }(\%)\end{array}$} & \multicolumn{3}{|c|}{$\begin{array}{l}\text { Number of crAss-like phage } \\
\text { contigs (greater than } 70 \mathrm{~kb} \text {; NR) }\end{array}$} & \multirow[t]{2}{*}{$\begin{array}{l}\text { Lysogeny-related genes } \\
\text { (\% crAss-like phages) }\end{array}$} \\
\hline & & $\begin{array}{l}\text { VLP- } \\
\text { DNA }\end{array}$ & $\begin{array}{l}\text { Total } \\
\text { Microbial- } \\
\text { DNA }\end{array}$ & Total & \\
\hline F3 & $0.1-14 \%$ & 22 & 14 & 36 & 30.5 \\
\hline F4 & $2-4 \%$ & 19 & 22 & 41 & 39 \\
\hline F5 & $4-10 \%$ & 19 & 16 & 35 & 40 \\
\hline F7 & $0.03-1.6 \%$ & 27 & 11 & 38 & 50 \\
\hline F11 & $0.001 \%$ & 5 & 4 & 9 & 44 \\
\hline F12 & $0.001 \%$ & 00 & 0 & 0 & NA \\
\hline F13 & $2-25 \%$ & 4 & 7 & 11 & 36 \\
\hline F14 & $0-0.2 \%$ & 10 & 4 & 14 & 35 \\
\hline F16 & $0.04-2 \%$ & 15 & 9 & 24 & 68.8 \\
\hline F18 & $0-1.8 \%$ & 8 & 6 & 14 & 28.6 \\
\hline F21 & $0.1-1 \%$ & 10 & 8 & 18 & 61 \\
\hline F29 & $3-10 \%$ & 5 & 10 & 15 & 26.7 \\
\hline
\end{tabular}

